\title{
Accurate and efficient computation of optical absorption spectra of molecular crystals: the case of the polymorphs of ROY
}

\author{
Joseph C. A. Prentice ${ }^{\dagger, \ddagger}$ and Arash A. Mostofi ${ }^{\ddagger}$ \\ $\dagger$ Department of Materials, University of Oxford, Parks Road, Oxford OX1 3PH, United \\ Kingdom \\ $\ddagger$ Department of Materials, Department of Physics, and the Thomas Young Centre for \\ Theory and Simulation of Materials, Imperial College London, London SW7 2AZ, United \\ Kingdom
}

\begin{abstract}
When calculating the optical absorption spectra of molecular crystals from first principles, the influence of the crystalline environment on the excitations is of significant importance. For such systems, however, methods to describe the excitations accurately can be computationally prohibitive due to the relatively large system sizes involved. In this work, we demonstrate a method that allows optical absorption spectra to be computed both efficiently and at high accuracy. Our approach is based on the spectral warping method successfully applied to molecules in solvent. It involves calculating the absorption spectrum of a supercell of the full molecular crystal using semi-local TDDFT, before warping the spectrum using a transformation derived from smallerscale semi-local and hybrid TDDFT calculations on isolated dimers. We demonstrate the power of this method on three polymorphs of the well known colour polymorphic compound ROY, and find that it outperforms both small-scale hybrid TDDFT dimer
\end{abstract}


calculations and large-scale semi-local TDDFT supercell calculations, when compared to experiment.

\section{Introduction}

Molecular crystals are a class of solid composed of individual molecules bound together by relatively weak intermolecular interactions, such as van der Waals forces. ${ }^{1}$ Molecular solids have been the subject of extensive interest over the years, thanks to their actual or potential applications in fields such as organic LEDs, ${ }^{2}$ ferroelectrics, ${ }^{3}$ pharmaceuticals, ${ }^{4}$ and solar cells. ${ }^{5}$ One of the key features of many molecular solids is polymorphism: the ability of the same compound to form several different structures, depending on the temperature, pressure, and other conditions. Importantly, this difference in structure can manifest itself in differences between the observable properties of the polymorphs; for example, different polymorphs of pharmaceutical compounds can vary in their pharmaceutical effectiveness, creating both challenges and opportunities for drug development. ${ }^{6}$

Among the most important properties of a molecular crystal, particularly for solar cells or similar applications, is its optical absorption. When different polymorphs of the same compound exhibit different optical absorption properties, this is known as colour polymorphism. ${ }^{7}$ Such compounds have several potential uses, ${ }^{8}$ including temperature or mechanical sensors, ${ }^{9,10}$ lasers, ${ }^{11}$ and organic field effect transistors. ${ }^{12}$ If the optical absorption properties of the polymorphs of a candidate compound can be predicted ahead of time, this can help direct experimental efforts towards more promising compounds, saving resources and accelerating discovery. One way these predictions can be made is by using first-principles materials modelling methods to describe the electronic structure, such as density functional theory (DFT). Extensive research efforts have been focused on predicting the crystal structures of

polymorphs of compounds ${ }^{13,14}$ using high-throughput workflows, allowing the structure of polymorphs to be predicted from first principles accurately and at reasonable computational 
cost. The question is then: can we do something similar for the optical absorption spectra of these compounds?

One of the most popular methods for calculating absorption spectra of molecular crystals is time-dependent density functional theory (TDDFT), thanks to its balance of accuracy and computational efficiency ${ }^{15,16}$ compared to other methods, such as many-body perturbation theory via the GW-BSE approach. ${ }^{17}$ Previous work on molecular crystals using TDDFT has largely focused on improving accuracy by using more complex density functionals, ${ }^{15,18-21}$ such as optimally-tuned screened range-separated hybrid (OT-SRSH) functionals, ${ }^{22,23}$ but such calculations can be prohibitively computationally expensive to perform routinely, especially as the system size increases.

A computationally efficient alternative is to calculate the optical properties of an extended system using computationally efficient, but less accurate, semi-local TDDFT, and to apply a suitable correction to the spectrum. Previous work has shown that the unphysical tendency of semi-local TDDFT to over-delocalise excitations can be counteracted by constraining the excitations to be localised in real space. ${ }^{16,24,25}$ This in turn necessitates the use of supercells to converge the real-space environment in which the excitation is embedded. As for the correction to the spectrum, this problem is similar to that faced in the calculation of optical absorption spectra of chromophores in solvent: long-range interactions between the chromophore and solvent can very strongly affect the absorption spectrum. ${ }^{24}$ To address this problem for solvated chromophores, Ge et al. introduced a method in which semi-local TDDFT calculations of a large system consisting of a chromophore in explicit solvent are corrected using hybrid TDDFT calculations on isolated chromophores (or chromophores interacting with only a few solvent molecules). ${ }^{26}$ This so-called spectral warping method ${ }^{\ddagger}$ has since been used successfully for a range of chromophores in different solvents. ${ }^{25}$

In this paper, we present a method, based on the spectral warping method of Ge et al., ${ }^{26}$ for accurately calculating the optical absorption properties of molecular crystals, in partic-

\footnotetext{
${ }_{\ddagger}^{\ddagger}$ Also known as colour morphing. Note that the spectral warping method referred to here should not be confused with the time domain transformation often used in electronics.
} 
ular colour polymorphs. Semi-local TDDFT calculations are performed on a large periodic supercell (referred to as 'semi-local crystal' calculations from here on), and a correction to the semi-local TDDFT results is then applied, derived from hybrid TDDFT calculations on dimers (referred to as 'hybrid dimer' calculations from here on). In this work, we refer to this method as the crystal spectral warping method. We demonstrate the power of the crystal spectral warping method by applying it to three polymorphs of the strongly colour polymorphic compound ROY, ${ }^{27}$ and show that it significantly outperforms semi-local crystal and hybrid dimer calculations in terms of predicting experimental spectra. Our crystal spectral warping approach also gives better agreement with experiment than TDDFT calculations on molecular crystal supercells using the PBE0 hybrid functional, whilst having significantly lower computational cost.

\section{The compound ROY}

One of the most famous examples of colour polymorphism is 5-methyl-2-[(2-nitrophenyl)amino]3-thiophenecarbonitrile, or ROY for short (see Fig. 1). When first described in 1995, five polymorphs were known, which displayed red, orange and yellow colours (hence the name ROY). ${ }^{29}$ By 2010, another five polymorphs had been discovered, ${ }^{30-33}$ making it one of the most polymorphic compounds known up to that point. ${ }^{27}$ Another polymorph was reported in $2019,{ }^{34}$ bringing the number to eleven: nine whose crystal structures have been found using X-ray diffraction (R, Y, ON, OP, YN, ORP, YT04, R05, and PO13), and two whose structures remain unknown (RPL and YT04). ${ }^{7,35}$

ROY's polymorphs exhibit differences in both molecular conformation, and in crystal packing. Each polymorph is typically named after its colour, and, if necessary, a distinguish-

ing characteristic (e.g., $\mathrm{N}$ for needle, $\mathrm{P}$ for plate) or the year it was found. The Y polymorph exhibits piezochromism. ${ }^{36}$

It is known experimentally that the most important predictor of the colour of a polymorph 


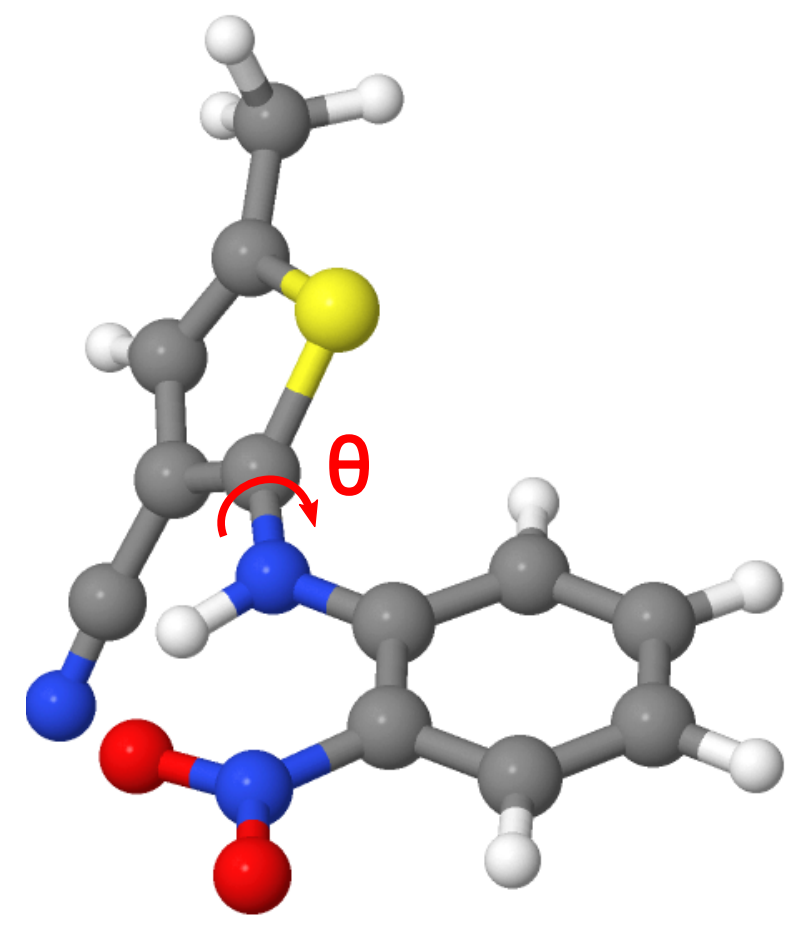

Figure 1: The molecular structure of the ROY molecule. H, C, N, O, and S atoms are white, grey, blue, red, and yellow respectively. Figure made with Jmol. ${ }^{28}$ Also labelled is the most important dihedral angle $\theta$, which controls how planar the molecule is, and is strongly correlated with the colour of the molecule.

of ROY is the angle $\theta$, which describes how planar the molecule is (see Fig. 1). Values of $\theta$ between approximately $20^{\circ}$ and $45^{\circ}$ correspond to red polymorphs, between $45^{\circ}$ and $60^{\circ}$ to orange polymorphs, and $60^{\circ}$ to $80^{\circ}$ to yellow polymorphs. ${ }^{7}$ Lower values of $\theta$ lead to more conjugation within the molecule, which affects the electronic states - most importantly, the states near the band edges - and thus the absorption spectrum. Computational work on ROY has mostly focused on establishing the relative stability of the polymorphs, ${ }^{7,37}$ rather than the absorption spectra. The only published first-principles calculations of the optical absorption properties of ROY (that we are aware of) considered single molecules extracted from the various polymorphs; ${ }^{38}$ the absorption spectra were calculated using several different methods, including TDDFT, and an empirical red-shift to account for the crystalline environment was applied, estimated from experimental data. In the present study, we go substantially beyond this, by not just considering the absorption due to isolated molecules, and by calculating the 
crystal red-shift from first principles.

We focus on three of the polymorphs of ROY - R, ON, and YN, between them representing the full range of colours present in ROY - as test cases for our method.

\section{Methods}

\subsection{Structures of the polymorphs}

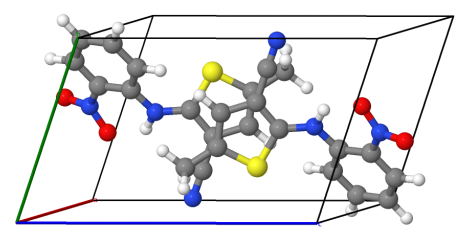

(a) $\mathrm{R}$

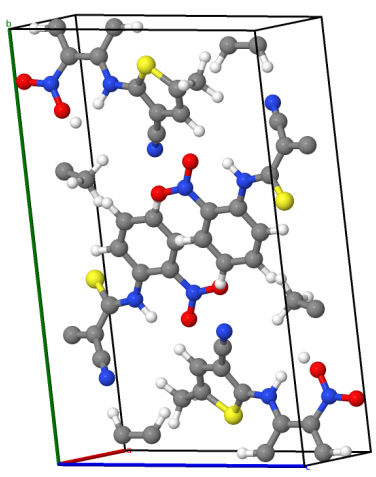

(b) $\mathrm{ON}$

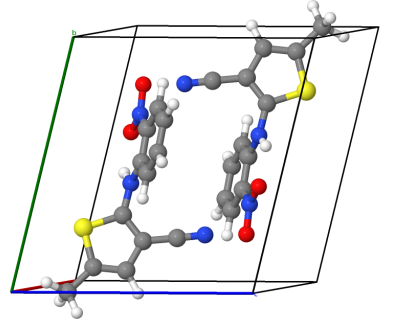

(c) $\mathrm{YN}$

Figure 2: The crystalline unit cells of the three polymorphs of ROY considered in this work. The $a, b$, and $c$ axes are coloured red, green, and blue respectively. H, C, N, O, and S atoms are white, grey, blue, red, and yellow respectively. Figures produced using Jmol. ${ }^{28}$

Three polymorphs of ROY are considered in this work: R, ON, and YN. YN and R both have $P \overline{1}$ symmetry and two molecular units in the unit cell, whilst $\mathrm{ON}$ has a higher $P 2_{1}$ symmetry and four molecular units in the unit cell. The structures are shown in Fig. 2. In the ON polymorph, each pair of nearest neighbour dimers is essentially equivalent, unlike in the YN and R polymorphs, where there are several different inequivalent nearest neighbour dimers - YN has two, and $\mathrm{R}$ has four.

Experimental structures were obtained from the Cambridge Structural Database, ${ }^{39}$ with the data originally sourced from Ref. 27. These experimental structures were all measured at or near room temperature. Experimental structural data from X-ray spectroscopy will typically contain some uncertainties, particularly in the positions of light elements; in contrast, 
however, larger-scale structural features, such as the lattice parameters or the important dihedral angle $\theta$, will be more accurately measured by such experiments. In order to reduce these errors, we optimise the experimental geometry using semi-local DFT, with lattice constants fixed at the experimental values. Fixing $\theta$ during the optimisation constitutes a non-linear constraint which is difficult to impose in practice - instead, for each polymorph we use an optimised geometry with as accurate a value of $\theta$ as possible, as discussed below.

Standard semi-local DFT does not include dispersion interactions, such as van der Waals forces, although these can be included easily using various semi-empirical schemes. Including dispersion interactions most strongly affects the lattice constants, through their effect on intermolecular binding energies. We can therefore implicitly include a large portion of the effect of dispersion interactions by fixing the lattice constants at their room temperature experimental values. This also facilitates comparison of our results with experimental measurements taken at room temperature. Using a dispersion correction scheme in our calculations should then provide small adjustments to our results, although since all schemes are approximate, these adjustments will come with errors too. These adjustments could lead to an optimised value of $\theta$ closer to the experimental value than by optimising the structure with semi-local DFT alone. As we cannot fix $\theta$ at its experimental value, we instead compare this to the value of $\theta$ obtained by relaxing the structures with and without the explicit inclusion of dispersion interactions, and select the structure with the value closest to experiment. This allows us to have a structure where the worst experimental errors have been relaxed away, but the important larger-scale parameters ( $\theta$ and the lattice parameters) are held at or close to their experimental values.

Here, dispersion interactions are explicitly included using the Tkatchenko-Scheffler (TS) scheme. ${ }^{40}$ The results of the relaxations (with experimental lattice constants fixed) both with and without the TS scheme can be found in Section S2 of the supporting information. We find that for the R and YN polymorphs, semi-local DFT alone gives the closest match to experiment, whilst for the ON polymorph, including dispersion interactions gives a better 
match to experiment. Because of this, we use the structure optimised with semi-local DFT only for the R and YN polymorphs, and the structure optimised with semi-local DFT + TS for the ON polymorph. These relaxed structures are the ones shown in Fig. 2.

\subsection{Calculation of absorption spectra}

As previously discussed, the crystal spectral warping method used in this work is an extension of the spectral warping method for calculating the absorption spectra of molecules in solvent. ${ }^{26}$ The spectral warping method is motivated by the fact that a hybrid functional is usually necessary to obtain quantitatively accurate optical absorption spectra from TDDFT calculations, but such calculations are often prohibitively computationally costly for large systems, such as molecules in explicit solvent, or supercells of a molecular crystal. Using a semi-local functional is much less computationally expensive for these systems, but is generally much less accurate. Nonetheless, the shape of the spectrum at low energies produced by a semi-local TDDFT calculation is often qualitatively similar to that obtained using a more accurate hybrid functional. The main differences are often a shift in the energies of the transitions and a rescaling of oscillator strengths. ${ }^{24,26,41}$ This implies that we should be able to apply a (series of) linear transformation(s) to the semi-local TDDFT spectrum, in order to obtain a good estimate of the hybrid TDDFT spectrum. This is known as spectral warping. For each excitation of interest, we can calculate the estimated hybrid excitation energy $\omega_{\text {hybrid }}$ from the semi-local excitation energy $\omega_{\text {semi-local via the transformation }}$

$$
\omega_{\text {hybrid }}=\alpha \omega_{\text {semi-local }}+\beta
$$

The oscillator strength $f$ is transformed in a similar way:

$$
f_{\text {hybrid }}=s f_{\text {semi-local }} \text {. }
$$


This observation is unhelpful, however, unless the parameters $\alpha, \beta$, and $s$ can be obtained without performing a large-scale (supercell) hybrid TDDFT calculation. The question is now how to obtain these parameters without performing such a calculation, so that the transformations in Equations (1) and (2) can be used to produce an approximate 'large-scale hybrid TDDFT' spectrum from a large-scale semi-local TDDFT spectrum.

Previous work on molecules in solvent has determined these parameters by making use of the fact that the excitations of interest are largely localised on a particular part of the system, i.e., the solute. This sub-system is small enough that we can perform semi-local and hybrid TDDFT calculations on it in isolation. By mapping the former calculation onto the latter, we can obtain values for $\alpha, \beta$ and $s$ for each relevant excitation. This mapping process is performed manually, by comparing the main orbitals involved in the excitations. These values for $\alpha, \beta$ and $s$ can then be inserted into Equations (1) and (2), which can then be used to separately warp each excitation of the larger system calculated using semilocal TDDFT, generating an absorption spectrum that approximates the hybrid TDDFT absorption spectrum of the larger system. This process, as used in this work, is shown schematically in Fig. 3. The advantage of this technique is that it includes the effects of the wider environment on the excitations, as well as treating the excitations accurately with hybrid functionals. We might expect that the absorption spectrum of a molecular crystal such as ROY will be dominated by effects arising from interactions between nearest neighbour molecules (i.e., molecular dimers), with the influence of more distant molecules providing a smaller screening correction. Calculations on molecular clusters of increasing size - presented in Section S7.2 of the supporting information - support this hypothesis. This suggests that the spectral warping method may work well for molecular crystals if we use the results for an isolated dimer to determine the warping parameters $\alpha, \beta$, and $s$. This dimer is 'cut out' of the molecular crystal, and used without any further geometry optimisation once it is isolated.

We compare the optical absorption spectra obtained by the crystal spectral warping 

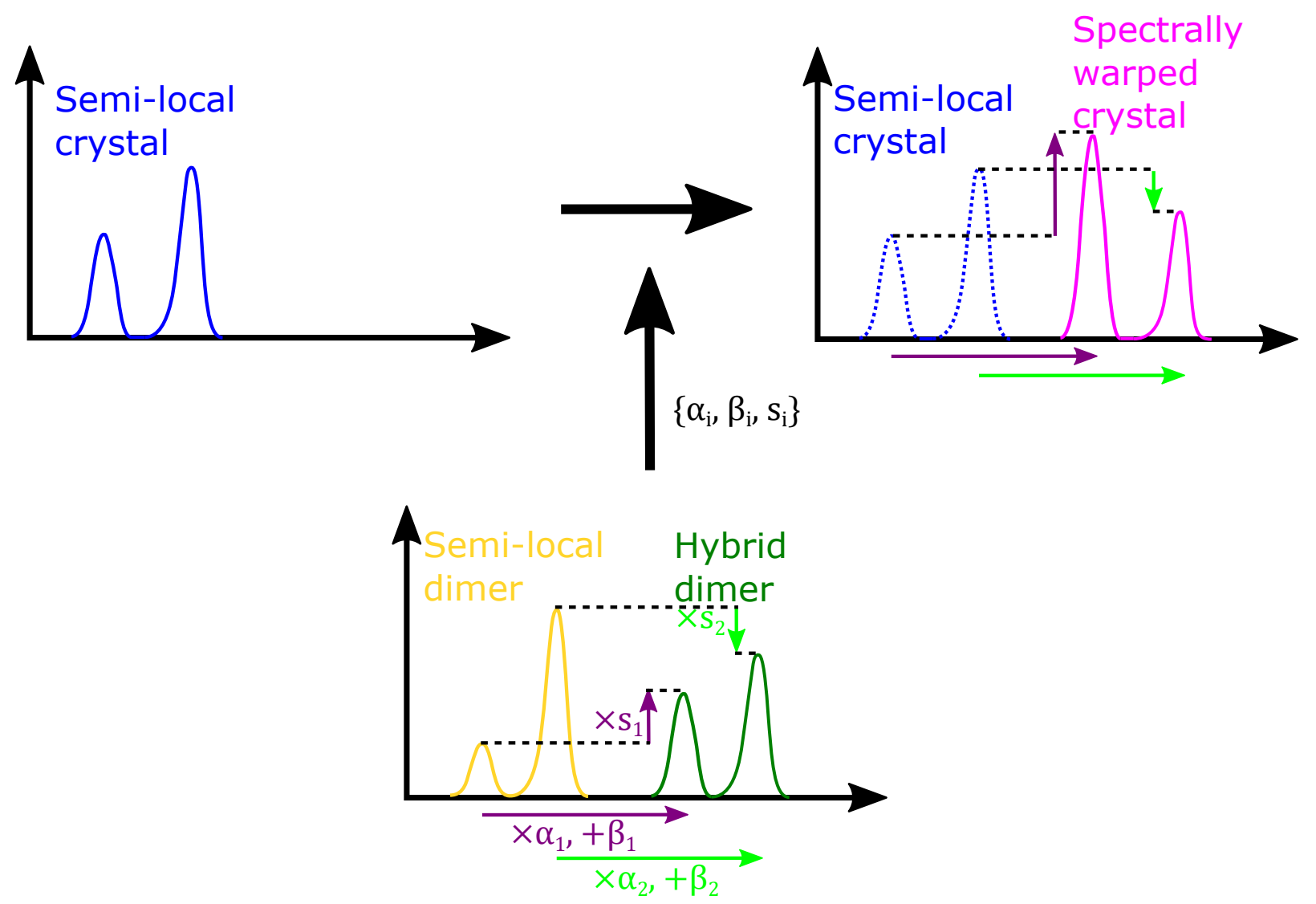

Figure 3: Schematic showing the crystal spectral warping process. By comparing semi-local dimer calculations (gold curve) and hybrid dimer calculations (dark green curve), we can determine linear transformations for the frequency and oscillator strength of each excitation (purple and light green arrows). We can then apply these transformations to semi-local crystal calculations (blue curve), producing a spectrally warped spectrum (magenta curve).

method to the spectra obtained through the semi-local crystal and hybrid dimer calculations outlined above. We also compare our results to experimental measurements. For the YN polymorph we also compare the results of the crystal spectral warping method to the results arising from applying a global hybrid functional (PBE0) directly to a supercell of the molecule crystal. Our reasons for using a global hybrid here are discussed in Section 3.3. A comparison to supercell TDDFT calculations using a non-optimally tuned range-separated hybrid functional are also presented in Section S3 of the supporting information. 


\subsection{Computational details}

The geometry optimisations discussed in Section 3.1 were performed with the plane-wave pseudopotential DFT code CASTEP ${ }^{42}$ using the PBE functional. ${ }^{43}$ As previously noted, dispersion interactions are included using the TS scheme ${ }^{40}$ for the ON polymorph only. These calculations were all run in periodic boundary conditions using only the unit cell of the polymorph in question. CASTEP's on-the-fly (OTF) norm-conserving pseudopotentials were used; the corresponding pseudopotential strings can be found in Section S1 of the supporting information. We used a kinetic energy cut-off of $1000 \mathrm{eV}$, and electronic kpoint sampling was performed in CASTEP using a Monkhorst-Pack grid ${ }^{44}$ with a spacing of $0.03 \AA^{-1}$.

The semi-local crystal TDDFT calculations, which also act as the base for the crystal spectral warping method, were performed with the code ONETEP, ${ }^{45}$ using the PBE functional. ${ }^{43}$ As semi-local TDDFT has a well-known issue with over-delocalisation, which leads to incorrect results, we enforce localisation by restricting the excitations to be localised on a given dimer, by truncating the response density kernel appropriately, similar to the approach used previous work. ${ }^{24}$ This is possible because ONETEP uses a set of localised non-orthogonal generalised Wannier functions (NGWFs) to represent the electronic structure. How appropriate dimers are selected is discussed in more detail below. The real-space constraint on the excitations means that a supercell must be used to converge the size of the real-space environment in which the excitation is embedded. We used a supercell consisting of $4 \times 3 \times 2$ unit cells (containing 48 molecular units) for the $\mathrm{YN}$ and $\mathrm{R}$ polymorphs, whilst a $4 \times 1 \times 2$ supercell (containing 32 molecular units) was used for the ON polymorph. Convergence calculations showed that the supercells used in this work were sufficiently large to obtain highly accurate results - for more details, see Section S7.1 of the supporting information. As a linear-scaling DFT code, ONETEP allows us to perform these large-scale calculations both efficiently and accurately. These calculations were performed in periodic boundary conditions. For consistency, we used the same pseudopotentials and kinetic en- 
ergy cut-off for our ONETEP calculations as those used in our CASTEP calculations. Unlike CASTEP, ONETEP only samples the $\Gamma$-point, as it is designed for use on large-scale systems (such as the supercells used here) where this is sufficient. More details of the calculational parameters used can be found in Section S1 of the supporting information.

The semi-local and hybrid dimer TDDFT calculations, which are used to obtain the parameters for the spectral warping transformations within the crystal spectral warping method, were performed using $\mathrm{NWChem}^{46}$ (unless noted), using the PBE and PBE0 ${ }^{47}$ functionals, respectively. We used PBE0 for this purpose as it has been shown to perform well in previous work on benchmarking different functionals for TDDFT in aromatic dye molecules, ${ }^{48}$ and also because it gives good results for band gaps in other molecular systems. ${ }^{49,50}$ PBE0 is also widely available and well-tested in most TDDFT codes. All NWChem calculations were performed in open boundary conditions, and the cc-pVDZ basis set was used for all species. This basis set does not include diffuse basis functions, which can substantially increase accuracy for some systems. ${ }^{51}$ However, we have found that including diffuse basis functions only produces a small rigid correction to our NWChem results, and that the spectral warping parameters are barely affected at all. This is due to the cancellation of systematic errors present in both the PBE and PBE0 calculations, which result in the calculations at different levels of theory shifting by almost exactly the same amount. As including diffuse basis functions significantly affects the computational expense and numerical stability of the calculations, ${ }^{51}$ we therefore use the cc-pVDZ basis set. Further discussion of the effects of using diffuse basis functions can be found in Section S7.3 of the supporting information.

The hybrid crystal TDDFT calculation for the YN polymorph was performed with the mixed Gaussian/plane-wave DFT code CP2K, ${ }^{52}$ using the hybrid functional PBE0. As previously noted, these calculations are significantly more expensive than equivalently-sized semi-local crystal TDDFT calculations, particularly in terms of computer memory. This limited us to performing these calculations on only one polymorph (YN), and also to a 
smaller supercell than that used elsewhere in the semi-local crystal TDDFT calculations $(3 \times 2 \times 1$ versus $4 \times 3 \times 2)$. These calculations were performed in periodic boundary conditions. We used the cc-TZV2P-GTH basis set for all species except S, for which we used TZV2P-GTH, and we used the potentials GTH-PBE-q1, -q4, -q6, -q5, and -q6 for H, $\mathrm{C}, \mathrm{O}, \mathrm{N}$, and $\mathrm{S}$, respectively. These potentials are those distributed with $\mathrm{CP} 2 \mathrm{~K}$. For hybrid functional calculations in periodic boundary conditions in $\mathrm{CP} 2 \mathrm{~K}$, it is necessary to limit the range of the exact-exchange part of the functional. For the PBE0 calculations, we used a truncated Coulomb potential with a cut-off radius of 5.8 bohr.

To reduce computational cost, the Tamm-Dancoff approximation ${ }^{53}$ (TDA) was applied in our ONETEP and CP2K TDDFT calculations. This approximation neglects the coupling between excitation and de-excitation processes, making the TDDFT eigenvalue problem Hermitian, and thus much easier to solve. The TDA typically gives accurate excitation frequencies, but performs worse for the oscillator strengths. ${ }^{16,54}$ In this work, we are primarily interested in the positions of the peaks in the absorption spectrum, so the TDA is a reasonable approximation to take. However, in our NWChem calculations, the TDDFT problem was solved in full, allowing any error in the oscillator strengths to be partially corrected by the spectral warping correction. Closer testing showed that using the TDA rather than full TDDFT for our NWChem calculations had very little effect on the spectral warping parameters, thanks to a cancellation of errors similar to that for diffuse basis functions described above, but we maintain the use of full TDDFT in our NWChem calculations for the sake of accuracy. Further discussion of the effects of using the TDA can be found in Section S7.4 of the supporting information.

One aspect of the calculations that should be particularly noted is that, as pointed out in Section 3.1, there are several inequivalent nearest neighbour dimers in the $\mathrm{YN}$ and $\mathrm{R}$ polymorphs. To build up a full spectrum, therefore, it is necessary to add together the contributions from each inequivalent dimer. This means the hybrid dimer results presented in Section 4 are actually the sum of the absorption spectra for each of these isolated dimers. 
Similarly, the semi-local crystal results presented are the sum of the absorption spectra with the excitation restricted to be on each of these dimers in turn. To obtain the crystal spectral warping results, the spectral warping procedure described above is applied to each dimer separately - the semi-local TDDFT calculation with the excitation restricted to a given dimer is warped using the vacuum results for the same dimer. The warped results for all dimers are then summed to give the crystal spectral warping spectra.

Apart from the codes used to perform the actual TDDFT calculations, we also used $c 2 \mathrm{x}^{55}$ to construct supercells, and $\mathrm{Jmol}^{28}$ and VESTA ${ }^{56}$ for visualisation purposes.

\section{Results}

Before presenting the results of the crystal spectral warping method, it is important to discuss the spectral warping process used. Figs. 4a, b, and c show the absorption spectra used to calculate the spectral warping transformations for the R, ON, and YN polymorphs respectively, i.e., the absorption spectra calculated for isolated dimers using PBE and PBE0, summed over all symmetry-inequivalent nearest neighbour dimers. It should be noted that the PBE0 results presented in Fig. 4 correspond to the hybrid dimer calculations shown in Fig. 5. To obtain realistic, smoothed spectra, we apply a Gaussian broadening to the raw TDDFT excitation excitations, with standard deviation of 0.002 hartree $(0.0544 \mathrm{eV})$. This

results in the vast majority of the broadening shown, as the excitation energies were largely similar for symmetry-inequivalent dimers. To decide on which excitations spectral warping should be applied to, we look at the PBE0 results, and identify any excitations within the visible range that have significant oscillator strength.

In the $\mathrm{R}$ and $\mathrm{YN}$ polymorphs, there is only one relevant excitation by these criteria, making the spectral warping process straightforward. In the ON polymorph, however, there is more than one potentially relevant excitation in both the PBE and PBE0 spectra, meaning we need to perform spectral warping separately on each of these excitations. In both the 


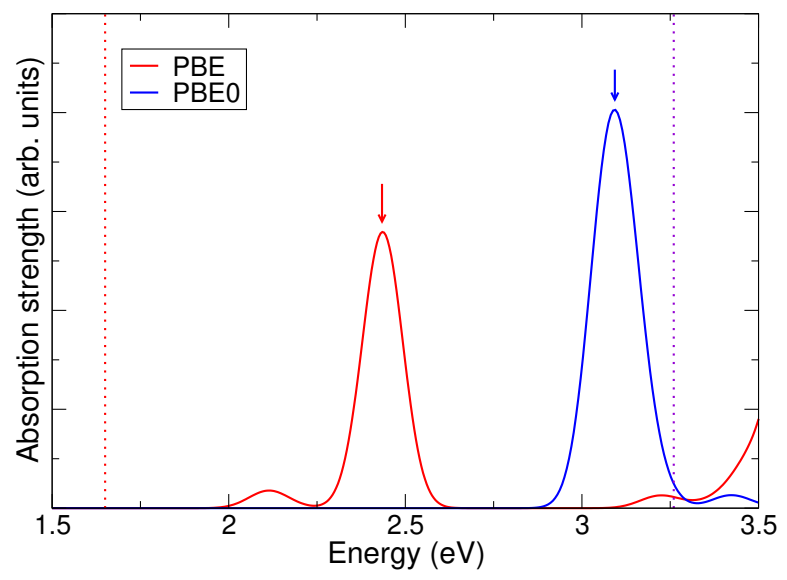

(a) $\mathrm{R}$

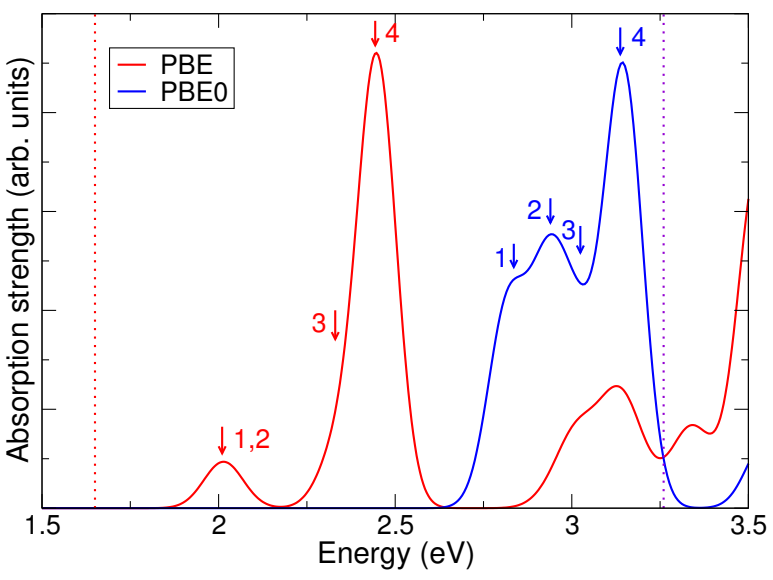

(b) $\mathrm{ON}$

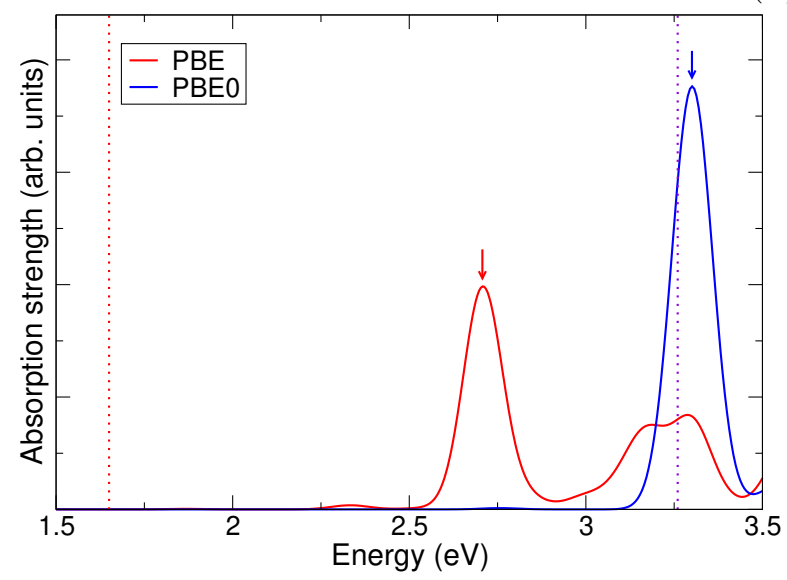

(c) $\mathrm{YN}$

Figure 4: Calculated absorption spectra for isolated dimers extracted from the R, ON, and YN polymorphs. Each subfigure shows the spectra calculated using both PBE and PBE0 functionals, summed over symmetry-inequivalent nearest neighbour dimers from each polymorph. These spectra correspond to the sum of the spectra used for the spectral warping process. Arrows indicate the peaks used to obtain the spectral warping parameters - in the case of $\mathrm{ON}$, corresponding peaks in the PBE and PBE0 spectra are labelled with the same number. The PBE0 results correspond to the hybrid dimer calculations discussed in the main text. The vertical dotted lines show the approximate edges of the visible spectrum.

PBE and PBE0 spectra, there are 4 peaks. In the PBE spectrum, peaks 1 and 2 have effectively the same energy, but in the PBE0 spectrum they split, as seen in Fig. 4b. Peak 4 is by far the strongest in both the PBE and PBE0 spectra, which means that the nearby peak 3 is not visible in Fig. 4b, although its approximate position is labelled. A spectral warping transformation is found for each corresponding pair of peaks: one mapping the ' 1 ' peaks onto each other, one mapping the '2' peaks onto each other, and so on. These spectral 


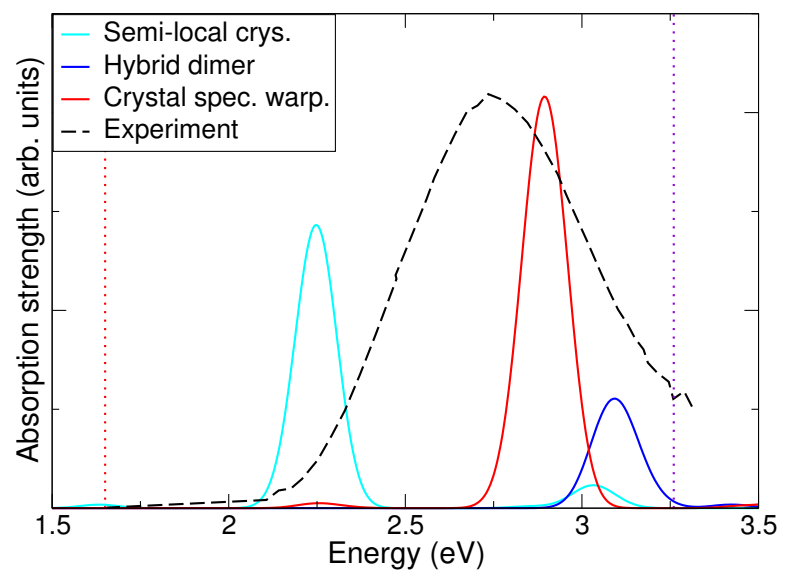

(a) $\mathrm{R}$

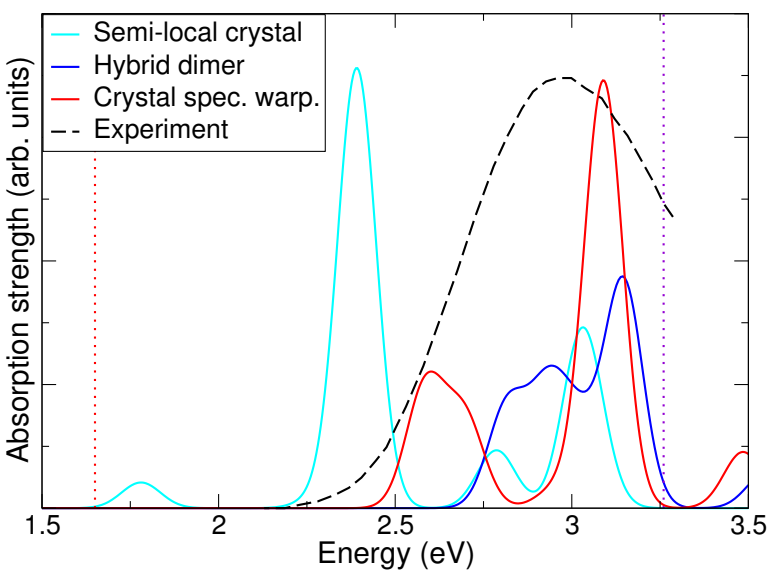

(b) $\mathrm{ON}$

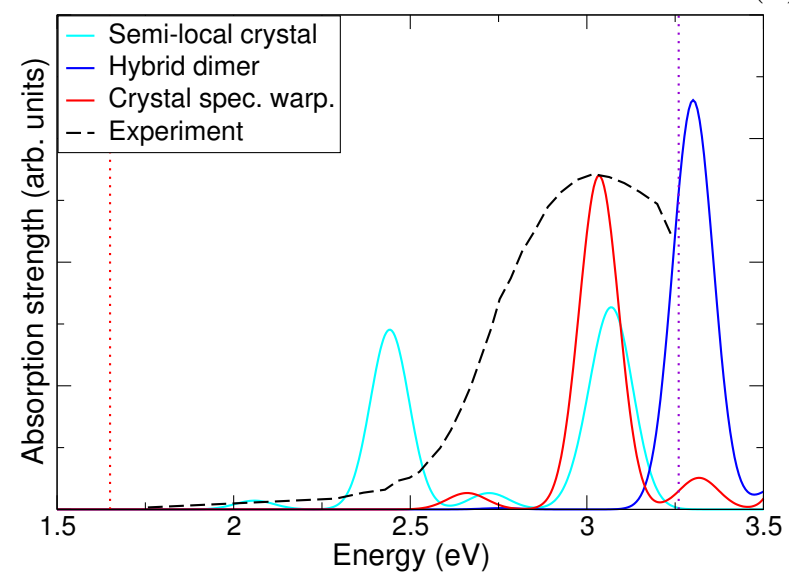

(c) $\mathrm{YN}$

Figure 5: Calculated absorption spectra for the R, ON, and YN polymorphs, calculated using several different methods, as described in Section 3.2. Each subfigure compares the spectra calculated by our crystal spectral warping method with the results of semi-local crystal and hybrid dimer calculations, as described in the main text, as well as experimental data, for each polymorph. The crystal calculations were performed using a $4 \times 3 \times 2$ supercell for the $\mathrm{R}$ and $\mathrm{YN}$ polymorphs, and a $4 \times 1 \times 2$ supercell for the ON polymorph. Experimental data is taken from Ref. 38. The vertical dotted lines show the approximate edges of the visible spectrum.

warping transformations are used to transform the semi-local crystal calculations for the ON polymorph. In all spectral warping calculations, we follow previous work ${ }^{24}$ and take $\alpha=1$, meaning that spectral warping effectively corresponds to a rigid frequency shift and a rescaling of the oscillator strength. The values of $\beta$ and $s$ resulting from this process are tabulated in Section S5 of the supporting information.

The absorption spectra calculated using the crystal spectral warping method for the R, 


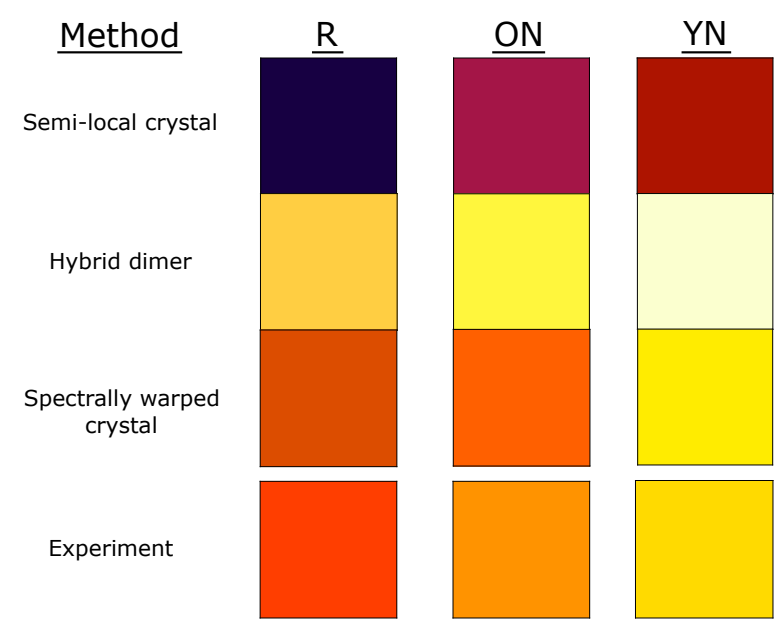

Figure 6: Swatches of colours generated from the absorption spectra calculated using several different methods, as well as from experimentally measured spectra. The spectra used are those shown in Fig. 5. The colour swatches were generated using the Colour Python package,${ }^{57}$ using the method described in Section $\mathrm{S} 4$ in the supporting information.

ON, and YN polymorphs are presented in Figs. 5a, b, and c respectively, and are compared to hybrid dimer results and experimental data. ${ }^{38}$ The same Gaussian broadening is applied as in Fig. 4. In the R and YN polymorphs, the crystal spectral warping method significantly improves on the hybrid dimer results, predicting a main absorption frequency much closer to the experimental value. For $\mathrm{R}$, the hybrid dimer calculations predict an excitation frequency $0.36 \mathrm{eV}$ above the experimental peak, whilst the crystal spectral warping method gives a frequency that is only $0.16 \mathrm{eV}$ above. For $\mathrm{YN}$, the hybrid dimer calculations predict a frequency that is $0.28 \mathrm{eV}$ above the experimental peak, but this difference is reduced by an order of magnitude by the crystal spectral warping method, giving a frequency only $0.02 \mathrm{eV}$ above experiment. In $\mathrm{ON}$, the picture is slightly more complicated due to the presence of four absorption peaks, which are likely smeared into one in the experimental measurement. However, if we look at the main (highest) peak (corresponding to peak 4 on Fig. 4b), we can see that the crystal spectral warping method agrees more closely with experiment than the hybrid dimer calculations. Hybrid dimer calculations predict an energy of $0.17 \mathrm{eV}$ away from the experimental peak for the main peak; using the crystal spectral warping method, the main peak is $0.11 \mathrm{eV}$ away. Importantly, the crystal spectral warping method is closer to 


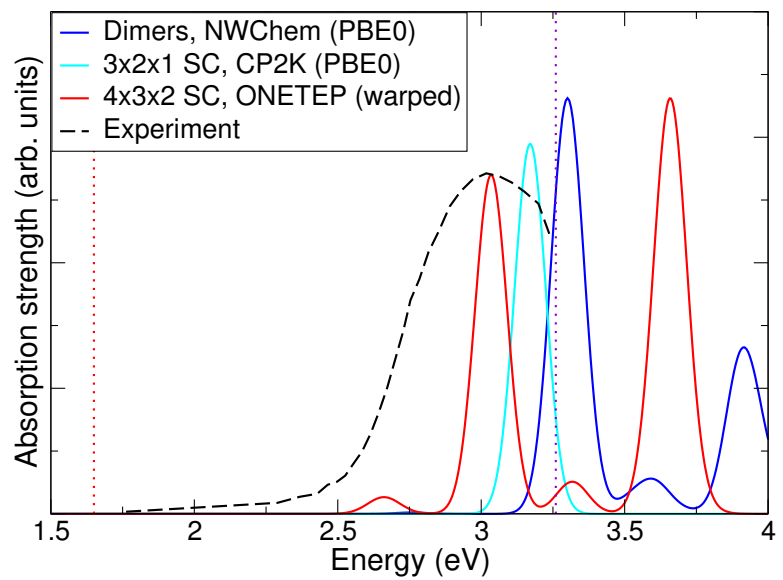

Figure 7: Absorption spectra for the YN polymorph calculated using several different methods, as described in Section 3.2. Selected results from Fig. 5c are compared to the results of hybrid TDDFT supercell calculations, performed with the PBE0 functional. ' $3 \times 2 \times 1$ $S C, C P 2 K$ (PBE0)' shows the spectra calculated with CP2K using PBE0 for a $3 \times 2 \times 1$ supercell. ' $4 \times 3 \times 2 S C$, ONETEP (warped)' and 'Dimers, NWChem (PBE0)' show the same data as 'Semi-local crystal' and 'Hybrid dimer' in Fig. 5c respectively, but have been renamed to emphasise the size of supercell and functional used. Experimental data is taken from Ref. 38. The vertical dotted lines show the approximate edges of the visible spectrum.

experiment than the hybrid dimer calculations in all polymorphs. More details of how the crystalline environment affects the character of the excitation are presented in Section S6 of the supporting information. Interestingly, these results suggest that, in the full crystalline environment, the excitations localise even further compared to in an isolated dimer. This suggests that the potential provided by the surrounding molecular crystal has the effect of confining the excitation, so that the excitation in the true molecular crystal is actually almost (but not completely) monomeric in nature.

The conclusion that the crystal warping method is an improvement over hybrid dimer calculations is backed up strikingly, although qualitatively, by generating colour swatches for each method used, as well as the experimental data. The Colour Python package ${ }^{57}$ is used to do this, following the method laid out in Section S4 of the supporting information. Fig. 6 shows these swatches for each polymorph. The swatches corresponding to the experimental data demonstrate that the three polymorphs are indeed red, orange, and yellow in colour, as expected. It can be seen that the spectral warping results give a colour significantly closer 
than the hybrid dimer results to the experimental colour. The hybrid dimer and crystal spectral warping methods give a yellow-orange and brown-red colour respectively for the $\mathrm{R}$ polymorph; for the ON polymorph, the colours are yellow and red-orange respectively; for the YN polymorph, the colours are light yellow and yellow respectively. This qualitative improvement in colour prediction is a direct result of the improvement shown in Fig. 5 and previously discussed. Alongside the quantitative data shown in Fig. 5, this demonstrates that the crystal spectral warping method, for a reasonable increase in computational cost, can significantly improve on the accuracy of the hybrid dimer calculations of absorption spectra and colour when compared to experiment.

In Fig. 7, we compare our crystal spectral warping method to the results of treating a crystal supercell of the YN polymorph fully with the hybrid functional PBE0, using CP2K. Again, the same Gaussian broadening is applied as in Fig. 4. As previously mentioned in Section 3.3, these hybrid crystal TDDFT calculations were performed on a smaller supercell than the semi-local crystal TDDFT calculations $(3 \times 2 \times 1$ versus $4 \times 3 \times 2)$. This should be borne in mind when comparing the results of the calculations, but the results still give an estimate of the relative accuracy of the crystal spectral warping method compared to full hybrid calculations, and also demonstrate the limits of what accuracy is achievable with limited computational time or hardware. The PBE0 functional performs reasonably well, predicting a frequency $0.16 \mathrm{eV}$ above the experimental peak, but the crystal spectral warping method still slightly outperforms it. This is likely due to the larger supercell size used in the semi-local ONETEP calculations, allowing slightly longer-range effects to be included in the calculation. This, coupled together with the significant reduction in cost, makes the crystal spectral warping method superior in this case. A further comparison against calculations using a non-optimally tuned version of the OT-SRSH functional ${ }^{22}$ is presented in Section S3 of the supporting information.

We also cross-check that the choice of code does not significantly affect accuracy, as both CP2K and ONETEP are used in this work for supercell calculations, by calculating 
the absorption spectrum of a $3 \times 2 \times 1$ supercell with both codes, using PBE and placing no localisation restriction on the excitations (results not shown). There is a less than $1 \%$ difference in the frequency of the strongest excitation, demonstrating that the choice of code does not affect the result.

\section{$5 \quad$ Potential future extensions}

The crystal spectral warping approach applied in this work is, at its core, quite a general one, and there are a number of different avenues through which it could be extended to include more complex effects. Although we do not do include these effects in the present work, in the following we discuss how the crystal spectral warping method could be modified to include them.

One phenomenon would be the effect of vibrations and temperature on the absorption spectrum. This is a topic that has been explored for chromophores in solvent with success. ${ }^{25,58}$ To include these effects, there are two main methods: the ensemble approach, and the Franck-Condon approach, although these can be employed simultaneously. The ensemble method typically includes the motion of the nuclei at a classical level, by averaging the absorption spectrum over a set of snapshots taken from a molecular dynamics trajectory. In a solvated small-molecule system, on the order of 100-1000 snapshots are usually required for convergence, ${ }^{25,58}$ to ensure that configurational space is sufficiently sampled. The number of snapshots required for a molecular crystal system such as ROY would require similarly careful convergence. Assigning and keeping track of symmetry-inequivalent dimers provides an additional complication, but likely only a minor one.

Whilst the ensemble method includes low-frequency, classical, vibrational contributions to the absorption spectrum, the Franck-Condon approach aims to include quantum mechanical vibronic effects, where electronic and vibrational degrees of freedom are excited simultaneously. ${ }^{58}$ This requires the computation of overlap integrals between nuclear wavefunctions in 
the ground and excited electronic states (under a set of simplifying assumptions), and therefore an optimised initial geometry. Combining the Franck-Condon and ensemble approaches naïvely, by computing overlap integrals for each snapshot, is likely to be unfeasible computationally, but work on solvated systems suggests that combining the zero-temperature Franck-Condon result with the ensemble approach gives good results. ${ }^{58}$

Another way to extend the crystal spectral warping method would be through using methods other than semi-local/hybrid TDDFT for the two levels of calculation. In particular, going beyond hybrid TDDFT could allow phenomena such as excitons to be included; such methods could include the Bethe-Salpeter equation, ${ }^{59}$ quantum Monte Carlo, ${ }^{60}$ or even (multi-reference) coupled cluster theory. ${ }^{61}$ However, there are three considerations regarding which methods could be picked.

Firstly, it must be computationally feasible to use the two levels of theory on the system sizes required of them - it must be feasible to apply the lower level of theory (semi-local TDDFT in this work) to both small- and large-scale systems (in this work, dimers and supercells respectively), and it must be feasible to apply the higher level of theory (hybrid TDDFT in this work) to small-scale systems.

Secondly, the lower level of theory must be trusted to give a qualitatively correct description of the system in both small- and large-scale systems. If this is not true of the lower level of theory, the crystal spectral warping method will not work, no matter which theory is used for the higher-level calculations.

Thirdly, mapping excitations computed at the lower level of theory to those computed at the higher level may become more complicated if the two methods are less similar than in this work - for example, if the lower level of theory is density-based, but the higher level is wavefunction-based. This is not a restriction, but does provide an additional complication that would need to overcome. 


\section{Conclusions}

In this work, we have presented the crystal spectral warping method, based on applying a simple warping to large-scale crystalline semi-local TDDFT calculations, to accurately predict the absorption spectra of molecular crystals. This method is adapted from a method previously used successfully for molecules in solvent. ${ }^{25,26}$ We have applied our crystal spectral warping method to three of the polymorphs of the highly colour polymorphic compound ROY, and compared its performance to a method based on hybrid TDDFT calculations on dimers, and a theoretically more accurate but significantly more computationally expensive full hybrid TDDFT method. The results of these comparisons show that the crystal spectral warping method improves on the hybrid dimer calculations, and also outperforms the full hybrid method, at least for the PBE0 hybrid functional. Our results also suggest that to calculate absorption spectra for molecular crystals, different methods will be appropriate in different scenarios, in order to balance accuracy and computational cost. If high accuracy is required, but computational resources are limited, the crystal spectral warping method presented here is likely to be the best option. If only an approximate result is required, smaller hybrid dimer TDDFT calculations may be more cost-effective.

This work presented here will allow significantly higher accuracy calculations of absorption spectra of molecular crystals than was possible previously, for a modest increase in computational expense, making such calculations routine. The crystal spectral warping method can also be easily implemented in a workflow, making it possible to automate the prediction of absorption spectra. This should enable high-throughput exploration of the optical absorption properties of molecular crystals and their polymorphs, making the search for systems with specific optical properties significantly more computationally efficient. 


\section{Supporting Information Available}

Further details of pseudopotentials and basis sets used in this work; discussion of the effect of dispersion corrections on structure; comparison of results for the YN polymorph against non-optimally tuned range-separated hybrid functional results; outline of colour prediction theory used in this work; further details of the spectral warping parameters used in this work; discussion of the character of the excitations in dimer and supercell geometries; analysis of approximations made, including supercell size convergence, use of dimer as a basic unit, Gaussian basis set convergence, and use of the Tamm-Dancoff approximation.

\section{Acknowledgement}

The authors acknowledge support from the UK Collaborative Computational Project for the Study of the Electronic Structure of Condensed Matter (CCP9) and the Engineering and Physical Sciences Research Council in the form of Software Infrastructure Grant $\mathrm{EP} / \mathrm{P} 02209 \mathrm{X} / 1$. The authors also acknowledge the support of the Thomas Young Centre for Theory and Simulation of Materials, through grant TYC-101. J.C.A.P. acknowledges the support of St Edmund Hall, University of Oxford, through the Cooksey Early Career Teaching and Research Fellowship. Via our membership of the UK's HPC Materials Chemistry Consortium, which is funded by EPSRC (EP/L000202), and the UKCP consortium, which is also funded by EPSRC (EP/P022561/1), this work used the UK Materials and Molecular Modelling Hub (MMM Hub) for computational resources, which is partially funded by EPSRC (EP/P020194/1 and EP/T022213). The authors are also grateful to the Imperial College Research Computing Service for computational resources. ${ }^{62}$ The authors would like to acknowledge the use of the University of Oxford Advanced Research Computing (ARC)

facility in carrying out this work. ${ }^{63}$ Finally, the authors would like to thank Dr. Rui Guo for drawing our attention to this interesting system. 


\section{References}

(1) Silinsh, E. A.; Capek, V. Organic Molecular Crystals: Interaction, Localization and Transport Phenomena; AIP Press: New York, 1994.

(2) Zou, S.-J.; Shen, Y.; Xie, F.-M.; Chen, J.-D.; Li, Y.-Q.; Tang, J.-X. Recent advances in organic light-emitting diodes: toward smart lighting and displays. Mater. Chem. Front. $2020,4,788$.

(3) Khatun, M.; Kabir, E.; Rahman, M. A review on polymer and organic ferroelectrics for flexible high energy storage material. AIP Conf. Proc. 2019, 2142, 040014.

(4) Duggirala, N. K.; Perry, M. L.; Almarsson, Ö.; Zaworotko, M. J. Pharmaceutical cocrystals: along the path to improved medicines. Chem. Commun. 2015, 52, 640.

(5) Zhou, R.; Jiang, Z.; Yang, C.; Yu, J.; Feng, J.; Adil, M. A.; Deng, D.; Zou, W.; Zhang, J.; Lu, K.; Ma, W.; Gao, F.; Wei, Z. All-small-molecule organic solar cells with over $14 \%$ efficiency by optimizing hierarchical morphologies. Nat. Commun. 2019, 10, 5393.

(6) Censi, R.; Di Martino, P. Polymorph Impact on the Bioavailability and Stability of Poorly Soluble Drugs. Molecules 2015, 20, 18759.

(7) Nogueira, B. A.; Castiglioni, C.; Fausto, R. Color polymorphism in organic crystals. Commun. Chem. 2020, 3, 1-12.

(8) Lu, B.; Liu, S.; Yan, D. Recent advances in photofunctional polymorphs of molecular materials. Chinese Chem. Lett. 2019, 30, 1908.

(9) Gentili, D.; Durso, M.; Bettini, C.; Manet, I.; Gazzano, M.; Capelli, R.; Muccini, M.; Melucci, M.; Cavallini, M. A time-temperature integrator based on fluorescent and polymorphic compounds. Sci. Rep. 2013, 3, 2581. 
(10) Li, M.; Zhang, Q.; Wang, J.-R.; Mei, X. Mechanochromism triggered fluorescent color switching among polymorphs of a natural fluorescence pigment. Chem. Commun. 2016, 52, 11288.

(11) Liao, Q.; Wang, X. G.; Lv, S.; Xu, Z.; Zhang, Y.; Fu, H. Cluster-Mediated Nucleation and Growth of J- and H-Type Polymorphs of Difluoroboron Avobenzone for Organic Microribbon Lasers. ACS Nano 2018, 12, 5359.

(12) Hsieh, C.-T.; Chen, C.-Y.; Lin, H.-Y.; Yang, C.-J.; Chen, T.-J.; Wu, K.-Y.; Wang, C.-L. Polymorphic Behavior of Perylene and Its Influences on OFET Performances. J. Phys. Chem. C 2018, 122, 16242.

(13) Price, S. L. Control and prediction of the organic solid state: a challenge to theory and experiment. Proc. Roy. Soc. Lond. A 2018, 474, 20180351.

(14) Beran, G. J. O. Modeling Polymorphic Molecular Crystals with Electronic Structure Theory. Chem. Rev. 2016, 116, 5567.

(15) Kronik, L.; Neaton, J. B. Excited-State Properties of Molecular Solids from First Principles. Annu. Rev. Phys. Chem. 2016, 67, 587.

(16) Zuehlsdorff, T. J.; Hine, N. D. M.; Spencer, J. S.; Harrison, N. M.; Riley, D. J.; Haynes, P. D. Linear-scaling time-dependent density-functional theory in the linear response formalism. The Journal of Chemical Physics 2013, 139, 064104.

(17) Ulpe, A. C.; Bredow, T. GW-BSE Calculations of Electronic Band Gap and Optical Spectrum of $\mathrm{ZnFe}_{2} \mathrm{O}_{4}$ : Effect of Cation Distribution and Spin Configuration. ChemPhysChem 2020, 21, 546.

(18) Fonari, A.; Sutton, C.; Brédas, J.-L.; Coropceanu, V. Impact of exact exchange in the description of the electronic structure of organic charge-transfer molecular crystals. Phys. Rev. B 2014, 90, 165205. 
(19) Bisti, F.; Stroppa, A.; Picozzi, S.; Ottaviano, L. Fingerprints of the hydrogen bond in the photoemission spectra of croconic acid condensed phase: An x-ray photoelectron spectroscopy and ab-initio study. J. Chem. Phys. 2011, 134, 174505.

(20) Leng, X.; Yin, H.; Liang, D.; Ma, Y. Excitons and Davydov splitting in sexithiophene from first-principles many-body Green's function theory. J. Chem. Phys. 2015, 143, 114501.

(21) Lüftner, D.; Refaely-Abramson, S.; Pachler, M.; Resel, R.; Ramsey, M. G.; Kronik, L.; Puschnig, P. Experimental and theoretical electronic structure of quinacridone. Phys. Rev. B 2014, 90, 075204.

(22) Manna, A. K.; Refaely-Abramson, S.; Reilly, A. M.; Tkatchenko, A.; Neaton, J. B.; Kronik, L. Quantitative Prediction of Optical Absorption in Molecular Solids from an Optimally Tuned Screened Range-Separated Hybrid Functional. J. Chem. Theory Comput. 2018, 14, 2919.

(23) Bhandari, S.; Cheung, M. S.; Geva, E.; Kronik, L.; Dunietz, B. D. Fundamental Gaps of Condensed-Phase Organic Semiconductors from Single-Molecule Calculations using Polarization-Consistent Optimally Tuned Screened Range-Separated Hybrid Functionals. J. Chem. Theory Comput. 2018, 14, 6287-6294.

(24) Zuehlsdorff, T. J.; Haynes, P. D.; Hanke, F.; Payne, M. C.; Hine, N. D. M. Solvent Effects on Electronic Excitations of an Organic Chromophore. J. Chem. Theory Comput. 2016, 12, 1853 .

(25) Zuehlsdorff, T. J.; Haynes, P. D.; Payne, M. C.; Hine, N. D. M. Predicting solvatochromic shifts and colours of a solvated organic dye: The example of nile red. J. Chem. Phys. 2017, 146, 124504.

(26) Ge, X.; Timrov, I.; Binnie, S.; Biancardi, A.; Calzolari, A.; Baroni, S. Accurate and 
Inexpensive Prediction of the Color Optical Properties of Anthocyanins in Solution. $J$. Phys. Chem. A 2015, 119, 3816.

(27) Yu, L. Polymorphism in Molecular Solids: An Extraordinary System of Red, Orange, and Yellow Crystals. Acc. Chem. Res. 2010, 43, 1257.

(28) Jmol: an open-source Java viewer for chemical structures in 3D. http://www.jmol.org/.

(29) Stephenson, G. A.; Borchardt, T. B.; Byrn, S. R.; Bowyer, J.; Bunnell, C. A.; Snorek, S. V.; Yu, L. Conformational and Color Polymorphism of 5-Methyl-2-[(2nitrophenyl)amino]-3-thiophenecarbonitrile. J. Pharm. Sci. 1995, 84, 1385.

(30) Yu, L.; Stephenson, G. A.; Mitchell, C. A.; Bunnell, C. A.; Snorek, S. V.; Bowyer, J. J.; Borchardt, T. B.; Stowell, J. G.; Byrn, S. R. Thermochemistry and Conformational Polymorphism of a Hexamorphic Crystal System. J. Am. Chem. Soc. 2000, 122, 585.

(31) Mitchell, C. A.; Yu, L.; Ward, M. D. Selective Nucleation and Discovery of Organic Polymorphs through Epitaxy with Single Crystal Substrates. J. Am. Chem. Soc. 2001, 123,10830 .

(32) Chen, S.; Guzei, I. A.; Yu, L. New Polymorphs of ROY and New Record for Coexisting Polymorphs of Solved Structures. J. Am. Chem. Soc. 2005, 127, 9881.

(33) Chen, S.; Xi, H.; Yu, L. Cross-nucleation between ROY polymorphs. J. Am. Chem. Soc. 2005, 127, 17439.

(34) Gushurst, K. S.; Nyman, J.; Boerrigter, S. X. M. The PO13 crystal structure of ROY. CrystEngComm 2019, 21, 1363.

(35) Tan, M.; Shtukenberg, A. G.; Zhu, S.; Xu, W.; Dooryhee, E.; Nichols, S. M.; Ward, M. D.; Kahr, B.; Zhu, Q. ROY revisited, again: the eighth solved structure. Faraday Discuss. 2018, 211, 477. 
(36) Harty, E. L.; Ha, A. R.; Warren, M. R.; Thompson, A. L.; Allan, D. R.; Goodwin, A. L.; Funnell, N. P. Reversible piezochromism in a molecular wine-rack. Chem. Commun. 2015, 51, 10608.

(37) Vasileiadis, M.; Kazantsev, A. V.; Karamertzanis, P. G.; Adjiman, C. S.; Pantelides, C. C. The polymorphs of ROY: application of a systematic crystal structure prediction technique. Acta. Cryst. B 2012, 68, 677 .

(38) Yu, L. Color Changes Caused by Conformational Polymorphism: OpticalCrystallography, Single-Crystal Spectroscopy, and Computational Chemistry. J. Phys. Chem. A 2002, 106, 544.

(39) Groom, C. R.; Bruno, I. J.; Lightfoot, M. P.; Ward, S. C. The Cambridge Structural Database. Acta. Cryst. B 2016, 72, 171.

(40) Tkatchenko, A.; Scheffler, M. Accurate Molecular Van Der Waals Interactions from Ground-State Electron Density and Free-Atom Reference Data. Phys. Rev. Lett. 2009, 102, 073005 .

(41) Malcıŏlu, O. B.; Calzolari, A.; Gebauer, R.; Varsano, D.; Baroni, S. Dielectric and thermal effects on the optical properties of natural dyes: a case study on solvated cyanin. J. Am. Chem. Soc. 2011, 133, 15425.

(42) Clark, S. J.; Segall, M. D.; Pickard, C. J.; Hasnip, P. J.; Probert, M. I. J.; Refson, K.; Payne, M. C. First principles methods using CASTEP. Z. Kristallog. 2005, 220, 567.

(43) Perdew, J. P.; Burke, K.; Ernzerhof, M. Generalized Gradient Approximation Made Simple. Phys. Rev. Lett. 1996, 77, 3865.

(44) Monkhorst, H. J.; Pack, J. D. Special points for Brillouin-zone integrations. Phys. Rev. B 1976, 13, 5188 . 
(45) Prentice, J. C. A.; Aarons, J.; Womack, J. C.; Allen, A. E. A.; Andrinopoulos, L.; Anton, L.; Bell, R. A.; Bhandari, A.; Bramley, G. A.; Charlton, R. J.; Clements, R. J.; Cole, D. J.; Constantinescu, G.; Corsetti, F.; Dubois, S. M.-M.; Duff, K. K. B.; Escartín, J. M.; Greco, A.; Hill, Q.; Lee, L. P.; Linscott, E.; O'Regan, D. D.; Phipps, M. J. S.; Ratcliff, L. E.; Serrano, Á. R.; Tait, E. W.; Teobaldi, G.; Vitale, V.; Yeung, N.; Zuehlsdorff, T. J.; Dziedzic, J.; Haynes, P. D.; Hine, N. D. M.; Mostofi, A. A.; Payne, M. C.; Skylaris, C.-K. The ONETEP linear-scaling density functional theory program. J. Chem. Phys. 2020, 152, 174111.

(46) Aprà, E.; Bylaska, E. J.; de Jong, W. A.; Govind, N.; Kowalski, K.; Straatsma, T. P.; Valiev, M.; van Dam, H. J. J.; Alexeev, Y.; Anchell, J.; Anisimov, V.; Aquino, F. W.; Atta-Fynn, R.; Autschbach, J.; Bauman, N. P.; Becca, J. C.; Bernholdt, D. E.; Bhaskaran-Nair, K.; Bogatko, S.; Borowski, P.; Boschen, J.; Brabec, J.; Bruner, A.; Cauët, E.; Chen, Y.; Chuev, G. N.; Cramer, C. J.; Daily, J.; Deegan, M. J. O.; Dunning, T. H.; Dupuis, M.; Dyall, K. G.; Fann, G. I.; Fischer, S. A.; Fonari, A.; Früchtl, H.; Gagliardi, L.; Garza, J.; Gawande, N.; Ghosh, S.; Glaesemann, K.; Götz, A. W.; Hammond, J.; Helms, V.; Hermes, E. D.; Hirao, K.; Hirata, S.; Jacquelin, M.; Jensen, L.; Johnson, B. G.; Jónsson, H.; Kendall, R. A.; Klemm, M.; Kobayashi, R.; Konkov, V.; Krishnamoorthy, S.; Krishnan, M.; Lin, Z.; Lins, R. D.; Littlefield, R. J.; Logsdail, A. J.; Lopata, K.; Ma, W.; Marenich, A. V.; Martin del Campo, J.; Mejia-Rodriguez, D.; Moore, J. E.; Mullin, J. M.; Nakajima, T.; Nascimento, D. R.; Nichols, J. A.; Nichols, P. J.; Nieplocha, J.; Otero-de-la Roza, A.; Palmer, B.; Panyala, A.; Pirojsirikul, T.; Peng, B.; Peverati, R.; Pittner, J.; Pollack, L.; Richard, R. M.; Sadayappan, P.; Schatz, G. C.; Shelton, W. A.; Silverstein, D. W.; Smith, D. M. A.; Soares, T. A.; Song, D.; Swart, M.; Taylor, H. L.; Thomas, G. S.; Tipparaju, V.; Truhlar, D. G.; Tsemekhman, K.; Van Voorhis, T.; Vázquez-Mayagoitia, Á.; Verma, P.; Villa, O.; Vishnu, A.; Vogiatzis, K. D.; Wang, D.; Weare, J. H.; Williamson, M. J.; Windus, T. L.; Woliński, K.; Wong, A. T.; Wu, Q.; Yang, C.; Yu, Q.; Zacharias, M.; 
Zhang, Z.; Zhao, Y.; Harrison, R. J. NWChem: Past, present, and future. J. Chem. Phys. 2020, 152, 184102.

(47) Adamo, C.; Barone, V. Toward reliable density functional methods without adjustable parameters: The PBE0 model. J. Chem. Phys. 1999, 110, 6158.

(48) Jacquemin, D.; Wathelet, V.; Perpète, E. A.; Adamo, C. Extensive TD-DFT benchmark: singlet-excited states of organic molecules. J. Chem. Theory Comput. 2009, 5, 2420.

(49) Fedorov, I. A. Structure and electronic properties of perylene and coronene under pressure: First-principles calculations. Comput. Mater. Sci. 2017, 139, 252.

(50) Fedorov, I. A. Pressure effect on the band structure and topological properties of the electron density of pyrene: First-principles calculations. Chem. Phys. 2019, 518, 8.

(51) Papajak, E.; Zheng, J.; Xu, X.; Leverentz, H. R.; Truhlar, D. G. Perspectives on basis sets beautiful: seasonal plantings of diffuse basis functions. J. Chem. Theory Comput. 2011, 7, 3027.

(52) Kühne, T. D.; Iannuzzi, M.; Del Ben, M.; Rybkin, V. V.; Seewald, P.; Stein, F.; Laino, T.; Khaliullin, R. Z.; Schütt, O.; Schiffmann, F.; Golze, D.; Wilhelm, J.; Chulkov, S.; Bani-Hashemian, M. H.; Weber, V.; Borštnik, U.; Taillefumier, M.; Jakobovits, A. S.; Lazzaro, A.; Pabst, H.; Müller, T.; Schade, R.; Guidon, M.; Andermatt, S.; Holmberg, N.; Schenter, G. K.; Hehn, A.; Bussy, A.; Belleflamme, F.; Tabacchi, G.; Glöß, A.; Lass, M.; Bethune, I.; Mundy, C. J.; Plessl, C.; Watkins, M.; VandeVondele, J.; Krack, M.; Hutter, J. CP2K: An electronic structure and molecular dynamics software package - Quickstep: Efficient and accurate electronic structure calculations. J. Chem. Phys. 2020, 152, 194103.

(53) Hirata, S.; Head-Gordon, M. Time-dependent density functional theory within the Tamm-Dancoff approximation. Chem. Phys. Lett. 1999, 314, 291. 
(54) Casida, M. E.; Huix-Rotllant, M. Progress in Time-Dependent Density-Functional Theory. Annu. Rev. Phys. Chem. 2012, 63, 287.

(55) Rutter, M. J. C2x: A tool for visualisation and input preparation for CASTEP and other electronic structure codes. Comput. Phys. Commun. 2018, 225, 174.

(56) Momma, K.; Izumi, F. VESTA 3 for three-dimensional visualization of crystal, volumetric and morphology data. J Appl Cryst 2011, 44, 1272.

(57) Colour Developers, Colour Manual. https://colour.readthedocs.io/en/latest/manual.html.

(58) Zuehlsdorff, T. J.; Isborn, C. M. Combining the ensemble and Franck-Condon approaches for calculating spectral shapes of molecules in solution. J. Chem. Phys. 2018, 148,024110 .

(59) Sagmeister, S.; Ambrosch-Draxl, C. Time-dependent density functional theory versus Bethe-Salpeter equation: an all-electron study. Phys. Chem. Chem. Phys. 2009, 11, 4451.

(60) Feldt, J.; Filippi, C. Quantum Chemistry and Dynamics of Excited States; John Wiley \& Sons, Ltd, 2020; pp 247-275.

(61) Serrano-Andrés, L.; Serrano-Pérez, J. J. In Handbook of Computational Chemistry; Leszczynski, J., Ed.; Springer Netherlands, 2012; pp 483-560.

(62) Imperial College Research Computing Service. 2018-2020; https://dx.doi.org/10. $14469 / \mathrm{hpc} / 2232$.

(63) University of Oxford Advanced Research Computing. 2021; http://dx.doi.org/10. $5281 /$ zenodo. 22558. 


\title{
Supporting Information for Accurate and efficient computation of optical absorption spectra of molecular crystals: the case of the polymorphs of ROY
}

\author{
Joseph C. A. Prentice and Arash A. Mostofi
}

July 5, 2021

\section{S1 Pseudopotentials and NGWFs}

The pseudopotential strings defining the pseudopotentials used in our CASTEP and ONETEP calculations were:

- $\mathrm{H}: 1|0.8| 14|16| 19 \mid 10 \mathrm{~N}(\mathrm{qc}=8)$

- $\mathrm{N}: 1|1.1| 23|26| 31 \mid 20 \mathrm{~N}: 21 \mathrm{~L}(\mathrm{qc}=9)$

- O: $1|1.2| 23|26| 31 \mid 20 \mathrm{~N}: 21 \mathrm{~L}(\mathrm{qc}=9)$

- S: $1|1.8| 6|7| 8 \mid 30 \mathrm{~N}: 31 \mathrm{~L}: 32 \mathrm{~N}$

ONETEP uses a basis set of localised non-orthogonal generalised Wannier functions (NGWFs) to describe the electronic structure. These functions are strictly localised, meaning they are zero outside a given radius from the atom they are associated with. This radius can be different for different atoms, and is a key parameter that controls the quality of the basis set, and thus the quality of the calculation. Different sets of NGWFs are used to describe the valence and conduction states, as conduction states are often more delocalised than valence states, and therefore require larger radii. In the calculations presented here, the radii of the valence NGWFs were chosen to be 8 bohr for $\mathrm{H}$ and 9 bohr for other species. The conduction NGWF radii were chosen to be 10 bohr for $\mathrm{H}$ and $11 \mathrm{bohr}$ for other species.

\section{S2 Inclusion of dispersion corrections}

In this work, we use experimental structures obtained using X-ray spectroscopy as a starting point. Such experimental measurements will likely produce accurate values for large-scale structural parameters of the system, such as the lattice 


\begin{tabular}{|c|cccc|}
\cline { 2 - 5 } \multicolumn{1}{c|}{} & \multicolumn{4}{c|}{$\theta\left(^{\circ}\right)$} \\
\hline Polymorph & Experiment $^{\mathrm{S1}}$ & $\mathrm{PBE}$ & $\mathrm{PBE}+\mathrm{TS}$ & $\mathrm{PBE}+\mathrm{TS}$ (latt. params) \\
\hline $\mathrm{R}$ & 34.5 & 31.5 & 30.6 & 30.0 \\
$\mathrm{ON}$ & 44.7 & 36.5 & 41.4 & 34.9 \\
YN & 58.8 & 58.7 & 62.7 & 55.9 \\
\hline
\end{tabular}

Table 1: Values of $\theta$ resulting from geometry optimisations with different levels of theory, as described in the main text. In the PBE and PBE+TS geometry optimisations, the lattice parameters are kept fixed at the value measured in experiment ${ }^{\mathrm{S} 1}$, with only atomic positions optimised. In the PBE+TS (latt. params) geometry optimisations, both the atomic positions and lattice parameters were optimised.

parameters or the dihedral angle $\theta$ defined in Fig. 1 in the main text; however, there will also likely be significant errors in smaller-scale features, such as the positions of light atoms (e.g. hydrogen). We would like to remove or reduce these errors, whilst fixing the values of important large-scale features at their experimental values. If the lattice parameters were the only large-scale parameters we wanted to fix in this way, this would be very simple - we could simply perform a semi-local DFT geometry optimisation, with the lattice parameters fixed at their experimental values. Using the experimental lattice parameters also allows us to implicitly include thermal expansion effects. However, fixing $\theta$ is more difficult, as this constitutes a non-linear constraint on the optimisation, which is very difficult to implement practically. Instead, we aim to get the value of $\theta$ as close as possible to experiment by comparing the result of optimising the structures with and without the explicit inclusion of dispersion interactions.

Including dispersion interactions, such as van der Waals forces, in geometry optimisations of molecular crystals will most strongly affect the lattice constants, as it affects how tightly molecules are bound together. This will then have an indirect effect of the value of $\theta$. By fixing the lattice constants at their experimental values, as we do in this work, we implicitly include a large part of this effect. A scheme for including dispersion interactions explicitly will then provide small corrections, indirectly affecting the value of $\theta$. We checked for such corrections by comparing the value of $\theta$ (as discussed in Section 2 in the main text) for four structures: the experimental structure, the PBE-optimised structure with fixed lattice parameters, the $\mathrm{PBE}+\mathrm{TS}$-optimised structure with fixed lattice parameters, and the PBE + TS-optimised structure with optimised lattice parameters. TS here refers to the Tkatchenko-Scheffler scheme for including dispersion interactions semi-empirically ${ }^{\mathrm{S} 2}$. The results can be found in Table 1. We can see that, when the lattice parameters are fixed at the experimental values, $\mathrm{PBE}$ alone provides a better match to experiment than $\mathrm{PBE}+\mathrm{TS}$ in two of the three polymorphs $-\mathrm{R}$ and YN. In the ON polymorph, PBE + TS gives a better match to experiment than $\mathrm{PBE}$ alone. One potential explanation for this result is the combination of the fact that explicitly including dispersion interactions is expected to only provide small corrections to the semi-local 
DFT result with experimental lattice parameters, alongside the fact that the TS scheme is a simple semi-empirical scheme, and thus will contain errors. If these errors are comparable in size to these small corrections, it is quite possible that using the TS scheme will actually give slightly worse agreement with experiment than semi-local DFT alone.

In all three polymorphs, $\theta$ is poorly predicted when the lattice parameters are optimised with PBE $+\mathrm{TS}$ too. Such an optimisation also changes the lattice parameters significantly - $a$ shrinks by $12.1 \%, 2.7 \%$, and $4.3 \%$ in $\mathrm{YN}$, $\mathrm{R}$, and ON respectively. This is likely mostly due to the fact that the experimental lattice parameters include the effect of thermal expansion, whereas the PBE $+\mathrm{TS}$ optimised values are effectively at zero temperature. Taking the $\mathrm{PBE}+\mathrm{TS}$ optimised values for the lattice parameters as an approximation for the true zero-temperature values, the change between these and the experimental room-temperature values imply linear thermal expansion coefficients of $4.59 \times 10^{-4}, 0.92 \times 10^{-4}$, and $1.50 \times 10^{-4} \mathrm{~K}^{-1}$ for $\mathrm{YN}, \mathrm{R}$, and $\mathrm{ON}$, respectively. These values are comparable to those seen in other molecular crystals ${ }^{\mathrm{S} 3}$, demonstrating that thermal expansion likely accounts for the majority of the difference between the experimental and $\mathrm{PBE}+\mathrm{TS}$ lattice parameters.

The results of Table 1 demonstrate that the $\mathrm{R}$ and YN polymorphs are best described using the PBE-optimised structure, whilst the ON polymorph is best described using the PBE+TS-optimised structure. They also show that ensuring the inclusion of thermal effects on the structure via fixing the lattice parameters at their experimental values is also important in obtaining an accurate description.

\section{S3 Comparison against (non-optimally tuned) OT- SRSH functional}

In addition to the comparison of the crystal spectral warping method against supercell PBE0 TDDFT calculations for the YN polymorph in Section 4 of the main text, we also compared our results against supercell hybrid TDDFT calculations using a non-optimally tuned version of the OT-SRSH functional defined in Ref. S4. As our aim is to compute the optical absorption spectrum both accurately and efficiently, we do not specifically tune the parameters of the OT-SRSH functional to the system in question (making the 'OT' part of the name a slight misnomer in this work). They were instead selected to be in the middle of the range appropriate for similar molecular crystal systems: $\alpha=0.2^{\mathrm{S} 4}, \beta=0.2444$, as $\alpha+\beta=1 / \epsilon_{r}$ and $n=\sqrt{\epsilon_{r}} \approx 1.5$ for this kind of molecular crystal ${ }^{\mathrm{S} 5 \mathrm{~S} 6}$, and $\omega=0.25 \mathrm{bohr}^{-1}$, as this lies in the middle of the range found in other molecular crystals ${ }^{\mathrm{S}}$. It is worth noting that $\alpha$ and $\beta$ here represent parameters of the OT-SRSH functional, ${ }^{\mathrm{S} 4}$ and not the parameters of the spectral warping transformation in Eq. (1) in the main text.

These 'OT'-SRSH calculations were performed in CP2K ${ }^{\mathrm{S} 7}$ using the same supercell size and parameters as the supercell PBE0 TDDFT calculations pre- 
sented in Section 4 in the main text. To implement the OT-SRSH functional in $\mathrm{CP} 2 \mathrm{~K}$, we included the following section in our input file:

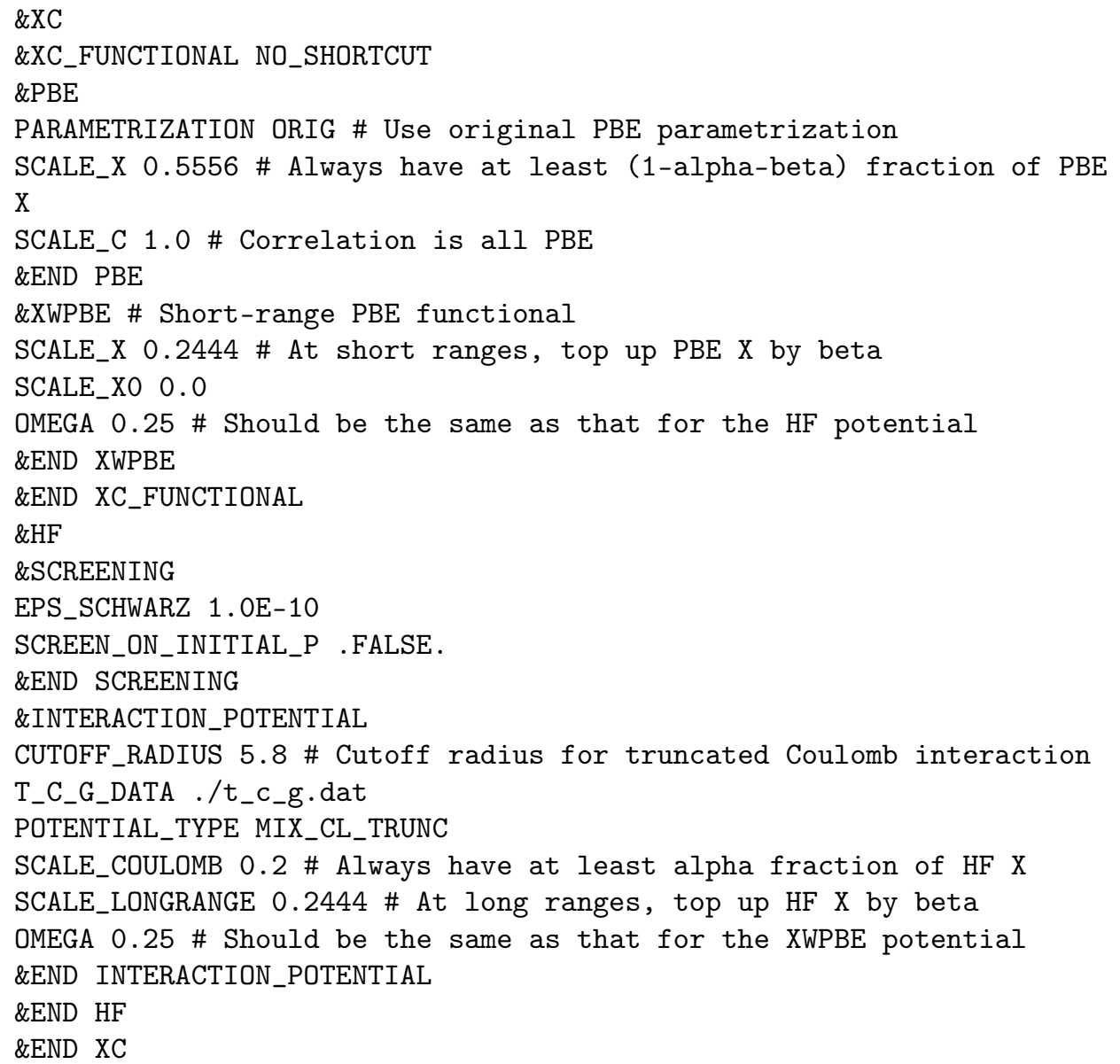

In Fig. S1, we additionally compare the results already presented in Fig. 7 in the main text to the results of treating a crystal supercell of the YN polymorph with the version of the OT-SRSH functional described above. The results do not compare particularly well against the crystal spectral warping method or supercell PBE0 TDDFT results, predicting a frequency $0.87 \mathrm{eV}$ above the experimental peak. This is likely because we have not actually optimally tuned the parameters of the OT-SRSH functional, but instead used values similar to those used in the literature. This suggests that, although OT-SRSH functionals are capable of exceptional accuracy, as in previous work ${ }^{\mathrm{S} 4}$, they may not be the best choice for general purpose calculations without first undergoing the tuning process. 


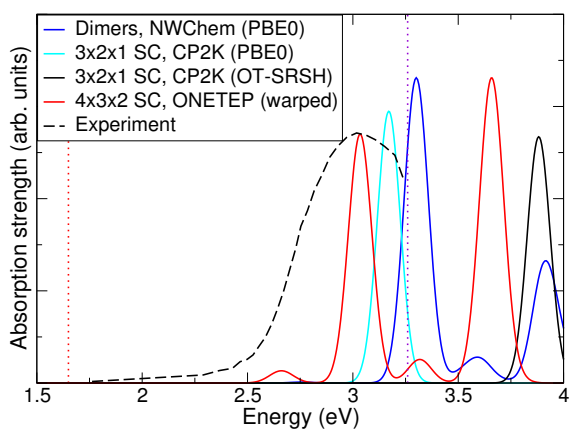

Figure S1: Absorption spectra for the YN polymorph calculated using several different methods. The data are the same as those presented in Fig. 7 in the main text, with the addition of the results of calculations performed with a non-optimally tuned version of the OT-SRSH functional. ' $3 \times 2 \times 1 S C, C P 2 K$ (PBE0)' and ' $3 \times 2 \times 1 S C, C P 2 K(O T-S R S H)$ ' show the spectra calculated with $\mathrm{CP} 2 \mathrm{~K}$ for a $3 \times 2 \times 1$ supercell, using the relevant functionals. ' $4 \times 3 \times 2 S C$, ONETEP (warped)' and 'Dimers, NWChem (PBE0)' show the same data as 'Semi-local crystal' and 'Hybrid dimer' in Fig. 5c in the main text respectively, but have been renamed to emphasise the size of supercell and functional used. Experimental data is taken from Ref. S8. The vertical dotted lines show the approximate edges of the visible spectrum.

\section{S4 Colour prediction theory}

Given an optical absorption spectrum, such as that produced by a TDDFT calculation, it is possible to calculate what colour a material with this absorption spectrum would appear as to the human eye. This can be done using tristimulus colorimetry theory $\mathrm{S} 9, \mathrm{~S} 10, \mathrm{~S} 11$. We first calculate the intensity of light transmitted through a sample of the material as

$$
I(\lambda)=I_{0}(\lambda) e^{-\alpha(\lambda) t},
$$

where $\alpha(\lambda)$ is the absorption spectrum from TDDFT as a function of wavelength $(\lambda), t$ is the thickness of the sample, and $I_{0}(\lambda)$ is the spectrum of the light source illuminating the sample. The response of the cone cells in the human retina to this spectrum must then be calculated. This is typically done by using the tristimulus colour-matching functions $x(\lambda), y(\lambda)$, and $z(\lambda)$ to obtain the colour indices $X, Y$, and $Z$ :

$$
\left(\begin{array}{l}
X \\
Y \\
Z
\end{array}\right)=N \int I(\lambda)\left(\begin{array}{l}
x(\lambda) \\
y(\lambda) \\
z(\lambda)
\end{array}\right) d \lambda .
$$

The $X Y Z$ colour indices can then be transformed to give $R G B$ indices, denoting the colour that would be observed. 


\begin{tabular}{|c|cccc|ccccc|cc|}
\hline Polymorph & \multicolumn{5}{|c|}{$\mathrm{R}$} & \multicolumn{4}{|c|}{ ON } & \multicolumn{3}{c|}{ YN } \\
\hline $\begin{array}{c}\text { Dimer } \\
\text { Excitation }\end{array}$ & 1 & 2 & 3 & 4 & \multicolumn{3}{c}{1} & & & 2 \\
\hline$\beta(\mathrm{meV})$ & 685 & 611 & 668 & 649 & 811 & 904 & 683 & 698 & 581 & 607 \\
$s$ & 1.12 & 1.73 & 1.69 & 1.37 & 6.60 & 12.04 & 2.56 & 0.97 & 1.90 & 2.12 \\
\hline
\end{tabular}

Table 2: Values of the spectral warping parameters $\beta$ and $s$, as defined in Equations (1) and (2) in the main text, used in this work. 'Dimer' labels the various symmetry-inequivalent dimers within each polymorph - spectral warping parameters are found for each dimer separately. 'Excitation' labels the (relevant) excitations seen in the absorption spectrum of each dimer - each excitation is spectrally warped separately. Only the ON polymorph has more than one relevant excitation in the absorption spectrum, and the labels used for the excitations in this case match those used in Fig. 4 in the main text.

In this work, we make use of the Colour Python package ${ }^{\mathrm{S} 12}$ to do the bulk of this computation - given the function $e^{-\alpha(\lambda) t}$ and the illuminant to use, it can compute a colour swatch, like those seen in Fig. 6 in the main text. We use the CIE standard D65 illuminant ${ }^{\mathrm{S} 13}$, meant to approximate daylight. For the generation of the function $e^{-\alpha(\lambda) t}$, we need to determine an appropriate thickness $t$. For consistency, we use the same thickness for the three calculated spectra for each polymorph; we also use the same thickness for all three experimental spectra. We take $t=\frac{A}{\bar{\alpha}_{\max }}$, where $\bar{\alpha}_{\max }$ is the mean of the maximum values of $\alpha(\lambda)$ of the three relevant spectra. This ensures that $\alpha(\lambda)$ is normalised close to the range $[0,1]$, but maintains the relative strength of the absorption peaks from different spectra. $A$ is a constant, which is taken to be 10 for the calculated spectra and 40 for the experimental spectra. In addition to this, to make the width of our calculated spectra resemble experimental spectra more closely, we apply a larger Gaussian broadening than seen in the main text in Figs. 4, 5, and 7. In these figures, a Gaussian broadening with standard deviation $0.0544 \mathrm{eV}$ was applied, so that separated peaks can be easily discerned. For the generation of the colour swatches, a broadening of $0.2177 \mathrm{eV}$ was applied to the $\mathrm{ON}$ and $\mathrm{YN}$ spectra, and a broadening of $0.3265 \mathrm{eV}$ was applied to the $\mathrm{R}$ spectra.

\section{S5 Spectral warping parameters}

Table 2 shows the spectral warping parameters $\beta$ and $s$, as defined by Equations (1) and (2) in the main text, as derived from dimer calculations, for each of the symmetry-inequivalent dimers in each of the polymorphs considered in this work. As mentioned in the main text, $\alpha$ is taken to be 1 throughout. 


\section{S6 Character of the excitations}

The effect of the crystalline environment on the character of the excitations can be investigated by considering the form of the response density on a given dimer for the strongest excitation in each polymorph, in both the isolated dimer and the crystalline environment. To allow an exact comparison to be made, both the crystalline and isolated dimer calculations were performed with ONETEP, using the PBE functional. Open boundary conditions were used for the dimer calculations. The results are shown in Fig. S2, which shows isosurfaces of the response density for the strongest excitation in each structure considered. In all three cases, the excitation changes its form significantly under the influence of the crystalline environment. The excitation becomes more localised onto one molecule, instead of being spread over both, and the contribution of the thiophenyl ring to the excitation increases (this is particularly pronounced in the $\mathrm{R}$ and $\mathrm{YN}$ results). This increasing localisation onto a single molecule may explain why single molecule calculations are able to qualitatively predict the ordering of the colours, as seen in Ref. S8.

\section{S7 Analysis of approximations made}

\section{S7.1 Supercell size convergence}

The crystal spectral warping method we apply in this work relies on excitations being localised on a small number of molecules - in the system investigated here, a dimer. Without restricting the excitations in this way, semi-local TDDFT would be unable to accurately describe the system due to unphysical delocalisation. However, restricting the excitations in a periodic TDDFT calculation also means that we need to consider how to avoid spurious interactions between the excitations and their periodic images, most likely through long-range electrostatic dipole-dipole interactions. This issue only arises if the excitation is restricted, and would be absent if the excitation is allowed to delocalise over the whole system. To avoid these spurious interactions, or at least make them small enough to neglect, it is necessary to use a supercell, so that an excitation and its periodic images are well separated.

Fig. S3 shows how the excitation spectrum of the YN polymorph, with excitations restricted to a particular dimer, changes as the size of the supercell increases from $3 \times 2 \times 1$ to $4 \times 3 \times 2$ to $5 \times 4 \times 3$. Using the $5 \times 4 \times 3$ supercell result as a reference, the excitation energies for the lower energy peak in the $3 \times 2 \times 1$ and $4 \times 3 \times 2$ calculations have an absolute error of $24 \mathrm{meV}$ and $2 \mathrm{meV}$ respectively, whilst the corresponding oscillator strengths have a relative error of $59 \%$ and $2 \%$ respectively. It is clear that both quantities, particularly the oscillator strengths, exhibit supercell size convergence. This shows, firstly, that the size of supercell used in the calculations affects the results significantly, and secondly, that the $4 \times 3 \times 2$ supercell provides an excellent level of accuracy for the YN polymorph. Because of this, in this work we use the $4 \times 3 \times 2$ supercell 
for the $\mathrm{R}$ and $\mathrm{YN}$ polymorphs, and the similarly sized $4 \times 1 \times 2$ supercell for the ON polymorph.

\section{S7.2 Use of dimer as basic unit}

One of the key assumptions of the (crystal) spectral warping method is that we can qualitatively describe the relevant excitations of the full (crystalline) system with a small isolated sub-system. This sub-system should be small enough that we are able to perform both semi-local and hybrid TDDFT on it, and thus calculate the spectral warping parameters required to correct semilocal TDDFT calculations performed on the full system. This is the process described in Section 3.2 of the main text. What this sub-system should include is, therefore, an important question.

If our excitations are largely molecular in nature (which they are in this work), the simplest sub-system to use would simply be a monomer - an isolated molecule. We can then go further, and consider the effect of molecular interactions on electronic excitations. To a first approximation, we would expect that interactions between nearest neighbour molecules would have the strongest effect on the excitations, suggesting using molecular dimers as the sub-system should improve the accuracy significantly. More distant molecules will likely have a smaller effect, suggesting that using molecular trimers and quadrimers will provide smaller corrections to the calculated electronic excitations.

To save computational expense, we would like to use the smallest sub-system that still provides an accurate qualitative description of the absorption spectrum. We can test this by performing calculations on several sub-system sizes, ranging from monomer to quadrimer, and analysing the results. Figs. S4a and b show such results for the $\mathrm{ON}$ and YN polymorphs respectively, calculated using the PBE0 functional. As in Figs. 4 and 5 in the main text, for the YN polymorph the dimer, trimer, and quadrimer spectra shown are actually a sum over symmetry-inequivalent dimers/trimers/quadrimers, normalised by the number of dimers/trimers/quadrimers included in the sum. The trimers and quadrimers considered were constructed by combining the symmetry-inequivalent dimers. The results clearly show that the dimer is the smallest sub-system that provides a qualitatively accurate description of the absorption spectrum for both polymorphs. In the ON polymorph, the dimer calculation correctly describes the multiple-peaked structure of the spectrum, with the energies of the peaks very close to the values in the larger quadrimer calculation. In the YN polymorph, although the dimer calculation is missing one or two small subsidiary peaks compared to the quadrimer calculation, it accurately describes the energy of the main absorption peak, which dominates the spectrum. In both polymorphs, using trimers or quadrimers only provides small corrections to the dimer results. This is consistent with these corrections being largely environmental screeninglike in nature. Since such effects are included in the crystal spectral warping method via the semi-local crystal TDDFT calculations, it is unnecessary to include them within the unit that excitations are localised on.

These results therefore suggest that dimers are an appropriate choice for the 
basic unit of our calculations, as they are the smallest sub-system that is able to provide a qualitatively correct result compared to larger clusters, whilst not including effects that will be included at another point in our calculations.

\section{S7.3 Use of cc-pVDZ basis set}

For all (TD)DFT calculations, it is important to ensure that the basis set used is able to accurately describe the Kohn-Sham wavefunctions of the system, and thus the density. In plane wave codes, such as CASTEP (and ONETEP indirectly), this can be done in a systematic way, by increasing the plane wave cut-off energy until the basis set is of sufficient size. For codes that use Gaussian basis sets, such as NWChem, this is more difficult. One way that Gaussian basis sets can be 'improved' in some cases is by the addition of diffuse basis functions, that extend further from the atoms than other basis functions. These can be useful for describing intermolecular interactions ${ }^{\mathrm{S} 16}$. However, including diffuse basis functions is often significantly more computationally expensive, not only because they directly increase the complexity of the calculation, but because they often cause problems with numerical stability. For this reason, it is better to avoid the use of diffuse basis functions unless required ${ }^{\mathrm{S} 16}$.

In this work, we use the cc-pVDZ basis set for all NWChem calculations, which does not include diffuse basis functions. There is also a version of this basis set with added diffuse basis functions, aug-cc-pVDZ. Investigating the effect of including these diffuse basis functions is important, as this could potentially affect the spectral warping parameters, and thus the crystal spectral warping result. It could also affect the hybrid dimer results that we compare our crystal spectral warping results against.

In order to compare the effect of including diffuse basis functions, Fig. S5 shows absorption spectra calculated using both the cc-pVDZ and aug-cc-pVDZ basis sets for the ON, and YN polymorphs, using the PBE and PBE0 functionals. The spectra are superpositions of the calculated spectra for symmetryinequivalent dimers, as in Figs. 4 and 5 in the main text. We only present results for two of the polymorphs because, as noted above, the aug-cc-pVDZ calculations are significantly more computationally expensive than the cc-pVDZ calculations. It can be seen that including diffuse basis functions lowers excitation energies by roughly $0.1 \mathrm{eV}$ in all cases, but that the form of the spectrum remains very similar. The rigidity of this shift means that the spectral warping shifts $\beta$ change by a mean of $13 \mathrm{meV}$ over both polymorphs, and a maximum of $33 \mathrm{meV}$. This shows that the spectral warping method is largely unaffected by the inclusion of diffuse basis functions.

The key point to note here is that the spectral warping parameters by looking at the difference between two TDDFT spectra, calculated at different levels of theory. Because of this, there is some degree of error cancellation, as errors that are present in both calculations (e.g. due to the neglect of diffuse basis functions) will cancel out. This allows accurate values for the spectral warping parameters to be calculated when the individual spectra may contain systematic errors. The results shown in Fig. S5 show that this is in fact the case here - 
although we have a small error in the cc-pVDZ spectra relative to the augcc-pVDZ spectra, this error almost entirely cancels out when calculating the spectral warping parameters. This demonstrates that using a basis set that doesn't include diffuse basis functions, such as cc-pVDZ, is sufficiently accurate for the purposes of this work. As this also significantly saves on computational expense, we choose to neglect diffuse basis functions throughout this work.

The effect of diffuse basis functions on the hybrid dimer results that the crystal spectral warping results are compared to is discussed in conjunction with the effect of the TDA in Section S7.4.

\section{S7.4 Use of Tamm-Dancoff approximation}

As discussed in the main text, the Tamm-Dancoff approximation (TDA) reduces the computational expense of TDDFT calculations, whilst typically maintaining accurate values for the excitation energies, although oscillator strengths can be less accurate. In this work, we use the TDA for all calculations performed with ONETEP and CP2K, whilst we use full TDDFT for all NWChem calculations.

In order to compare the relative accuracy of the TDA and full TDDFT methods, Fig. S6 shows absorption spectra calculated using both the TDA and full TDDFT for the R, ON, and YN polymorphs, using the PBE and PBE0 functionals. The spectra are superpositions of the calculated spectra for symmetryinequivalent dimers, as in Figs. 4 and 5 in the main text. It can be seen that using the TDA raises excitation energies by roughly $0.1 \mathrm{eV}$ compared to full TDDFT in all cases, but that the form of the spectrum remains very similar. The rigidity of this shift means that the spectral warping shift changes little the average change is $25 \mathrm{meV}$ across all polymorphs, and the maximum change is $45 \mathrm{meV}$. This suggests that the spectral warping method is largely unaffected by whether TDA or full TDDFT is used. There is a cancellation of errors, similar to that described in Section S7.3 for diffuse basis functions, that means that both the TDA and full TDDFT give very similar values for the spectral warping parameters.

\section{References}

S1 Lian Yu. Polymorphism in molecular solids: An extraordinary system of red, orange, and yellow crystals. Acc. Chem. Res., 43:1257, 2010.

S2 Alexandre Tkatchenko and Matthias Scheffler. Accurate molecular Van der Waals interactions from ground-state electron density and free-atom reference data. Phys. Rev. Lett., 102:073005, 2009.

S3 Hsin-Yu Ko, Robert A. DiStasio, Biswajit Santra, and Roberto Car. Thermal expansion in dispersion-bound molecular crystals. Phys. Rev. Materials, 2:055603, 2018.

S4 Arun K. Manna, Sivan Refaely-Abramson, Anthony M. Reilly, Alexandre Tkatchenko, Jeffrey B. Neaton, and Leeor Kronik. Quantitative prediction 
of optical absorption in molecular solids from an optimally tuned screened range-separated hybrid functional. J. Chem. Theory Comput., 14:2919, 2018.

S5 S. De Jong, F. Groeneweg, and F. Van Voorst Vader. Calculation of the refractive indices of molecular crystals. J. Appl. Crystallogr., 24:171, 1991.

S6 Dylan Jayatilaka, Parthapratim Munshi, Michael J. Turner, Judith A. K. Howard, and Mark A. Spackman. Refractive indices for molecular crystals from the response of X-ray constrained Hartree-Fock wavefunctions. Phys. Chem. Chem. Phys., 11:7209, 2009.

S7 Thomas D. Kühne, Marcella Iannuzzi, Mauro Del Ben, Vladimir V. Rybkin, Patrick Seewald, Frederick Stein, Teodoro Laino, Rustam Z. Khaliullin, Ole Schütt, Florian Schiffmann, Dorothea Golze, Jan Wilhelm, Sergey Chulkov, Mohammad Hossein Bani-Hashemian, Valéry Weber, Urban Borštnik, Mathieu Taillefumier, Alice Shoshana Jakobovits, Alfio Lazzaro, Hans Pabst, Tiziano Müller, Robert Schade, Manuel Guidon, Samuel Andermatt, Nico Holmberg, Gregory K. Schenter, Anna Hehn, Augustin Bussy, Fabian Belleflamme, Gloria Tabacchi, Andreas Glöß, Michael Lass, Iain Bethune, Christopher J. Mundy, Christian Plessl, Matt Watkins, Joost VandeVondele, Matthias Krack, and Jürg Hutter. CP2K: An electronic structure and molecular dynamics software package - Quickstep: Efficient and accurate electronic structure calculations. J. Chem. Phys., 152:194103, 2020 .

S8 Lian Yu. Color changes caused by conformational polymorphism: Opticalcrystallography, single-crystal spectroscopy, and computational chemistry. J. Phys. Chem. A, 106:544, 2002.

S9 T. J. Zuehlsdorff, P. D. Haynes, M. C. Payne, and N. D. M. Hine. Predicting solvatochromic shifts and colours of a solvated organic dye: The example of nile red. J. Chem. Phys., 146:124504, 2017.

S10 O. B. Malcıŏlu, A. Calzolari, R. Gebauer, D. Varsano, and S. Baroni. Dielectric and thermal effects on the optical properties of natural dyes: a case study on solvated cyanin. J. Am. Chem. Soc., 133:15425, 2011.

S11 Xiaochuan Ge, Iurii Timrov, Simon Binnie, Alessandro Biancardi, Arrigo Calzolari, and Stefano Baroni. Accurate and inexpensive prediction of the color optical properties of anthocyanins in solution. J. Phys. Chem. A, 119:3816, 2015.

S12 Colour Developers. Colour Manual. https://colour.readthedocs.io/en/latest/manual.html.

S13 J. Schanda, editor. Colorimetry: Understanding the CIE system. J. Wiley \& Sons, Hoboken, NJ, 2007.

S14 K. Momma and F. Izumi. VESTA 3 for three-dimensional visualization of crystal, volumetric and morphology data. J Appl Cryst, 44:1272, 2011. 
S15 E. Aprà, E. J. Bylaska, W. A. de Jong, N. Govind, K. Kowalski, T. P. Straatsma, M. Valiev, H. J. J. van Dam, Y. Alexeev, J. Anchell, V. Anisimov, F. W. Aquino, R. Atta-Fynn, J. Autschbach, N. P. Bauman, J. C. Becca, D. E. Bernholdt, K. Bhaskaran-Nair, S. Bogatko, P. Borowski, J. Boschen, J. Brabec, A. Bruner, E. Cauët, Y. Chen, G. N. Chuev, C. J. Cramer, J. Daily, M. J. O. Deegan, T. H. Dunning, M. Dupuis, K. G. Dyall, G. I. Fann, S. A. Fischer, A. Fonari, H. Früchtl, L. Gagliardi, J. Garza, N. Gawande, S. Ghosh, K. Glaesemann, A. W. Götz, J. Hammond, V. Helms, E. D. Hermes, K. Hirao, S. Hirata, M. Jacquelin, L. Jensen, B. G. Johnson, H. Jónsson, R. A. Kendall, M. Klemm, R. Kobayashi, V. Konkov, S. Krishnamoorthy, M. Krishnan, Z. Lin, R. D. Lins, R. J. Littlefield, A. J. Logsdail, K. Lopata, W. Ma, A. V. Marenich, J. Martin del Campo, D. Mejia-Rodriguez, J. E. Moore, J. M. Mullin, T. Nakajima, D. R. Nascimento, J. A. Nichols, P. J. Nichols, J. Nieplocha, A. Otero-de-la Roza, B. Palmer, A. Panyala, T. Pirojsirikul, B. Peng, R. Peverati, J. Pittner, L. Pollack, R. M. Richard, P. Sadayappan, G. C. Schatz, W. A. Shelton, D. W. Silverstein, D. M. A. Smith, T. A. Soares, D. Song, M. Swart, H. L. Taylor, G. S. Thomas, V. Tipparaju, D. G. Truhlar, K. Tsemekhman, T. Van Voorhis, Á. Vázquez-Mayagoitia, P. Verma, O. Villa, A. Vishnu, K. D. Vogiatzis, D. Wang, J. H. Weare, M. J. Williamson, T. L. Windus, K. Woliński, A. T. Wong, Q. Wu, C. Yang, Q. Yu, M. Zacharias, Z. Zhang, Y. Zhao, and R. J. Harrison. NWChem: Past, present, and future. J. Chem. Phys., 152:184102, 2020.

S16 E. Papajak, J. Zheng, X. Xu, H. R. Leverentz, and D. G. Truhlar. Perspectives on basis sets beautiful: seasonal plantings of diffuse basis functions. J. Chem. Theory Comput., 7:3027, 2011. 


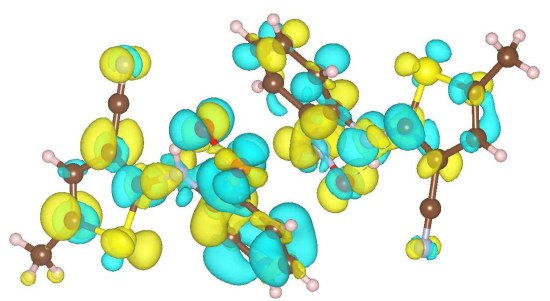

(a) R Dimer

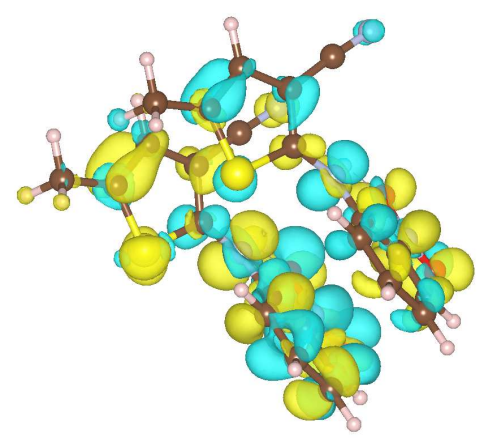

(c) ON Dimer

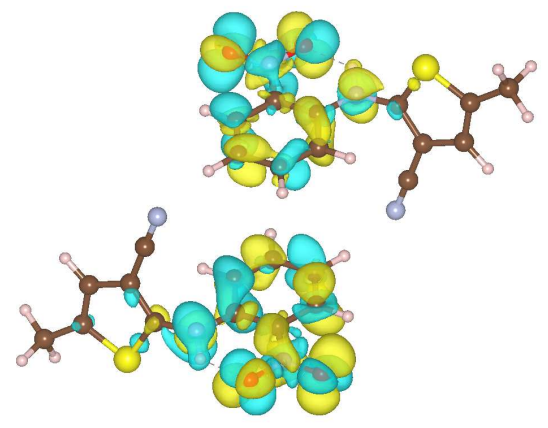

(e) YN Dimer

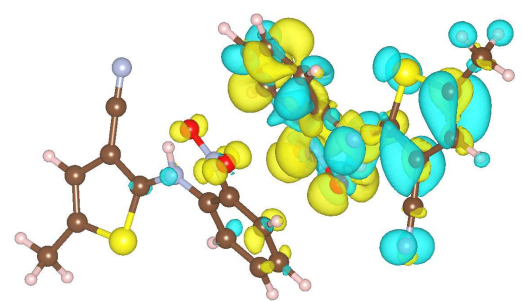

(b) R $4 \times 3 \times 2$ supercell

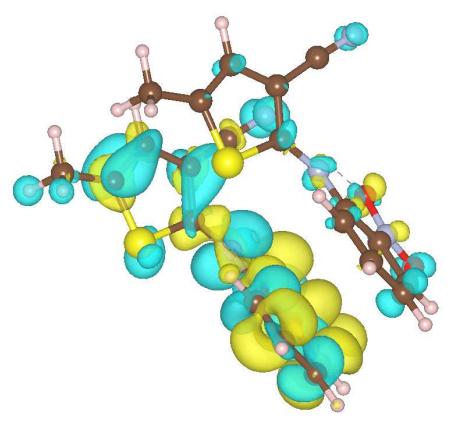

(d) ON $4 \times 1 \times 2$ supercell

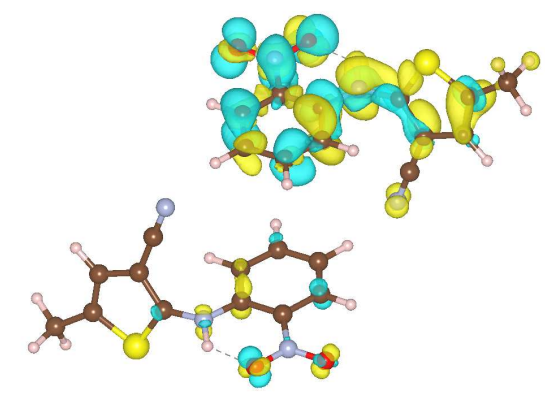

(f) YN $4 \times 3 \times 2$ supercell

Figure S2: Form of the strongest excitation in the R, ON and YN structures, calculated with ONETEP using PBE, as an isolated dimer or in a crystalline environment. The results for $\mathrm{R}$ for the dimer and crystalline environments are shown in (a) and (b) respectively, for ON in (c) and (d), and for YN in (e) and (f) respectively. The figures show an isosurface of the response density $\left(|n|=0.005 \mathrm{e} \AA^{-3}\right)$, with yellow and blue representing positive and negative response density values respectively. This isosurface is superimposed on the structure of the molecule. H, C, N, O, and S atoms are white, grey, blue, red, and yellow respectively. Figures produced using VESTA ${ }^{\mathrm{S} 14}$. 


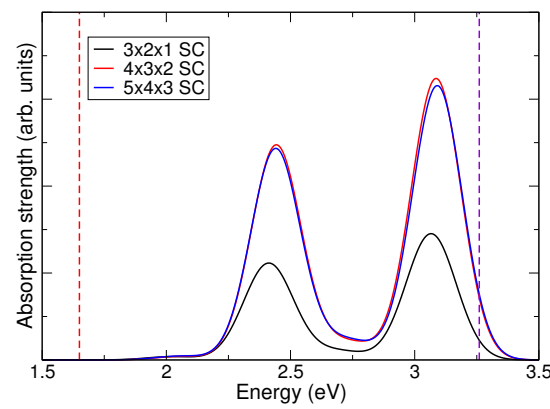

Figure S3: Comparison of the calculated absorption spectrum using supercells of increasing size $(3 \times 2 \times 1,4 \times 3 \times 2$, and $5 \times 4 \times 3)$, for the YN polymorph. The calculations were performed using the PBE functional with ONETEP. The spectra shown are not a sum over symmetry-inequivalent dimers, as described in the main text, but are all calculated with the excitations restricted to be on the same dimer. The Tamm-Dancoff approximation was applied for all calculations. The vertical dotted lines show the approximate edges of the visible spectrum.

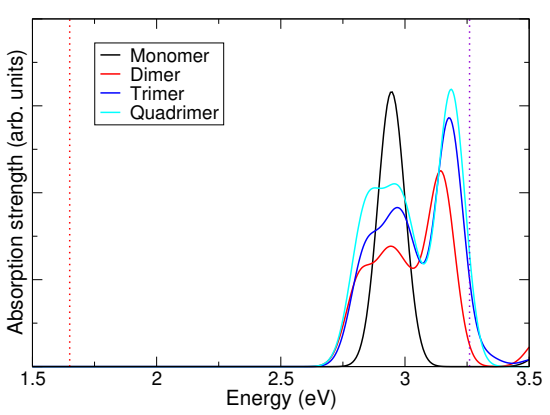

(a) $\mathrm{ON}$

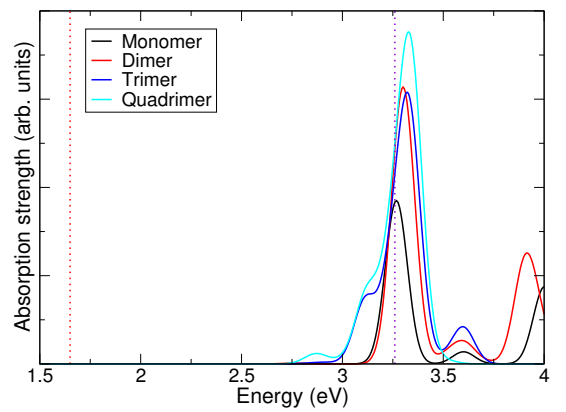

(b) $\mathrm{YN}$

Figure S4: Comparison of the calculated absorption spectrum for isolated molecular clusters of increasing size, for the ON and YN polymorphs. The clusters included are monomers, dimers, trimers, and quadrimers, containing 1, 2, 3, and 4 molecules respectively. The spectra shown are a sum over the spectra calculated for symmetry-inequivalent clusters, constructed by combining the symmetry-inequivalent dimers discussed in the main text. The results are calculated with NWChem ${ }^{\mathrm{S} 15}$ using the PBE0 functional and the cc-pVDZ basis set. The Tamm-Dancoff approximation was not applied. The vertical dotted lines show the approximate edges of the visible spectrum. 


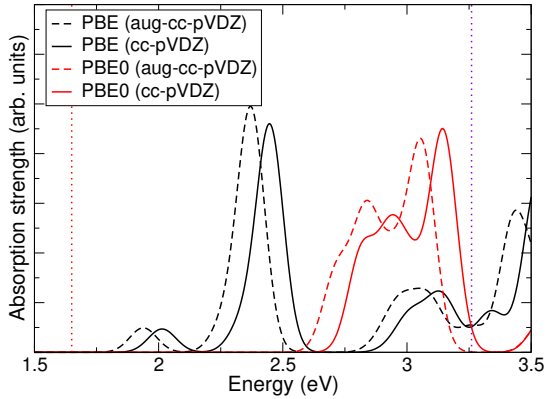

(a) $\mathrm{ON}$

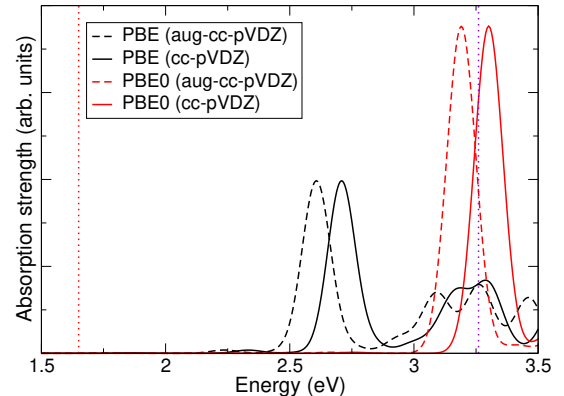

(b) $\mathrm{YN}$

Figure S5: Comparison of the dimer absorption spectrum, calculated using the cc-pVDZ basis set used in the rest of this work, and the aug-cc-pVDZ basis set, which includes diffuse functions, for the ON and YN polymorph. The spectra shown are a sum over symmetry-inequivalent dimers, as described in the main text. The results are calculated with $\mathrm{NWChem}^{\mathrm{S} 15}$ using the PBE and PBE0 functionals. The Tamm-Dancoff approximation was not applied. The vertical dotted lines show the approximate edges of the visible spectrum. 


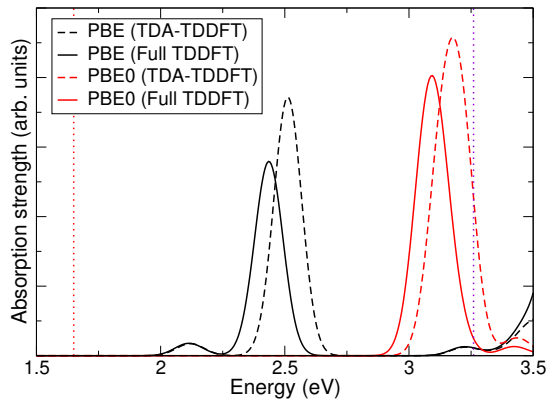

(a) $\mathrm{R}$

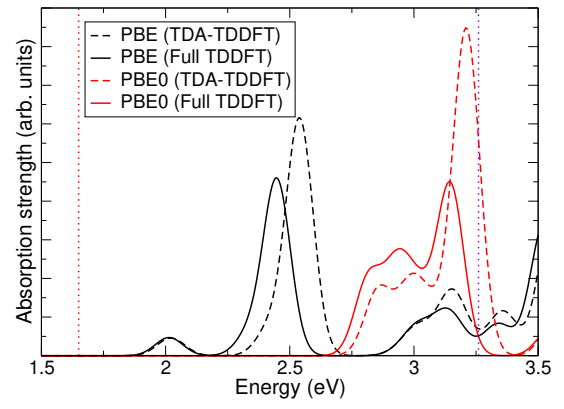

(b) $\mathrm{ON}$

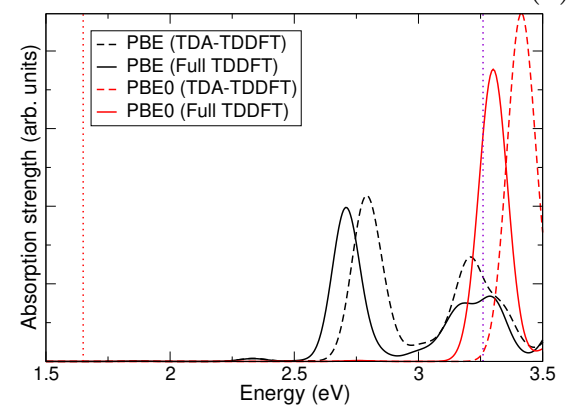

(c) YN

Figure S6: Comparison of the dimer absorption spectrum, calculated with and without the Tamm-Dancoff approximation, for the R, ON, and YN polymorphs. The spectra shown are a sum over symmetry-inequivalent dimers, as described in the main text. The results are calculated with $\mathrm{NWChem}^{\mathrm{S} 15}$ using the PBE and PBE0 functionals and the cc-pVDZ basis set. The vertical dotted lines show the approximate edges of the visible spectrum. 